\title{
L'HOMME L'Homme
}

Revue française d'anthropologie

$206 \mid 2013$

Varia

\section{L'oubli et le retour}

Figures d'une épopée mémorielle sur la Route de l'Esclave au Bénin

Oblivion and Return. Figures in a Memorial Epic on the Slave Route in Benin

\section{Gaetano Ciarcia}

\section{(2) OpenEdition}

\section{Journals}

Édition électronique

URL : http://journals.openedition.org/lhomme/24518

DOI : $10.4000 /$ lhomme. 24518

ISSN : 1953-8103

Éditeur

Éditions de l'EHESS

Édition imprimée

Date de publication : 4 juin 2013

Pagination : 89-119

ISSN : 0439-4216

Référence électronique

Gaetano Ciarcia, «L'oubli et le retour », L'Homme [En ligne], 206 | 2013, mis en ligne le 03 juin 2015, consulté le 01 mai 2019. URL : http://journals.openedition.org//homme/24518 ; DOI : 10.4000/ Ihomme.24518 


\title{
L'oubli et le retour
}

\section{Figures d'une épopée mémorielle sur la Route de l'Esclave au Bénin}

\section{Gaetano Ciarcia}

"Le non-oubli est un fantôme"

Nicole Loraux (1997: 165).

\begin{abstract}
A
BÉNIN MÉRIDIONAL, le lancement en 1994 de l'itinéraire intercontinental de la Route de l'Esclave, réalisé sous l'égide de la Conférence générale de l'Unesco, s'est focalisé sur l'idée d'un "patrimoine commun immatériel » de la traite des Noirs partagé par les peuples africains, amérindiens et européens. Soutenu par l'Organisation mondiale du tourisme, ce projet avait comme objectifs principaux: l'identification, la restauration et la promotion des sites, des bâtiments et des lieux de mémoire relevant de l'histoire de l'esclavage, afin de valoriser le développement économique et social grâce au tourisme culturel qu'elles étaient en mesure d'induire ${ }^{1}$.

En déjouant des amnésies intentionnelles, en réveillant des souvenirs enfouis, le lancement de la Route de l'Esclave a permis, entre autres effets sociaux majeurs, d'interroger et de scruter des mémoires jusqu'alors silencieuses, défaillantes ou rétives. L'institution d'un "circuit-mémorial » de la traite dans la ville côtière de Ouidah, située à quarante kilomètres à l'ouest de la capitale économique Cotonou, est intervenue également dans un contexte où se manifestait un clivage entre la reconstitution officielle de la période de la traite transatlantique et la perception intime du passé de l'esclavage local dans les concessions familiales ou les plantations de palmiers à huile. Il s'agit, en effet, d'un espace partagé entre l'affirmation politique des identités du présent et la reconstruction éthique des faits du passé. Si les mémoires les plus intimes de la condition d'esclave d'autrefois sont une "calebasse fermée " ${ }^{2}$ contenant des "secrets " généalogiques qui, la plupart du temps, peuvent à peine être murmurés, ce type de rétention
\end{abstract}

1. Unesco, résolution $27 \mathrm{C} / 13.13,27^{\mathrm{e}}$ session de la Conférence générale de l'Unesco, 1993.

2. La formulation est de l'un de mes interlocuteurs béninois. 
ou de dénégation est maintenant confronté à l'injonction qui est faite publiquement de commémorer le phénomène historique de l'esclavage, à travers l'évocation du rôle joué par les commerçants négriers européens et américains ou de celui des lignages esclavagistes locaux. Cette thématique se mêle de manière inextricable au traitement officiel des narrations à transmettre aux nouvelles générations et à "échanger " avec les destinataires/partenaires potentiels d'une mémoire culturelle en chantier : agences internationales du développement, touristes, chercheurs, cinéastes et photographes, visiteurs issus des "diasporas" afro-américaines, investisseurs, etc.

\section{Une route de fictions, entre oublis et retours}

Menant du centre de la ville de Ouidah - qui, d'après les estimations de l'historien Robin Law (2004), fut le port négrier le plus important d'Afrique, après celui de Luanda en Angola, en nombre d'esclaves y ayant transité, soit un million - à la plage, la Route de l'Esclave est à la fois un programme international et un parcours en terre battue, long d'un peu plus de trois kilomètres qui se compose en six étapes principales: la place de la Vente aux enchères - dite aussi place Chacha du surnom de Felix Francisco de Souza, le plus connu de tous les négriers d'Afrique, dont le pouvoir et les richesses en tant quallié du roi Guézo d'Abomey ont pris dans les mémoires locales une dimension légendaire ${ }^{3}$; l'Arbre de l'Oubli ; la case zomaï; le mémorial dans le village de Zoungbodji ; l'Arbre du Retour; la Porte du Non-Retour ${ }^{4}$. Conçus comme emblématiques de l'histoire de la traite à Ouidah, ces lieux sont reliés par vingt-deux sculptures aux styles différents qui jalonnent le trajet. Un tel aménagement devrait rappeler à la fois : la souffrance des captifs; la dimension sacrée des cultes vodun; la puissance et le prestige des souverains d'Abomey ayant dominé le territoire de Ouidah, après l'avoir arraché, en 1727, à la dynastie "autochtone» huéda, jusqu'à la colonisation française en 1892. Ainsi, mêlant matériaux, compositions plastiques et diverses images de la sujétion, du pouvoir, de la douleur, de la vie religieuse et profane, ces sculptures inscrivent l'histoire locale dans une sorte d'arène commémorative où le souvenir du passé de l'esclavage est associé à la domination des rois du Danhomé ayant précédé (et résisté à) la colonisation française. À cet égard, il faut préciser que les statues commémorant les monarques

3. Ce personnage a inspiré le roman de Bruce Chatwin, Le Vice-Roi de Ouidah (Paris, Grasset, 1982 [1980]), ainsi que le film réalisé par Werner Herzog, Cobra verde (1987).

4. Je reprends ici en partie une description que j'ai déjà présentée dans « Restaurer le futur : sur la Route de l'Esclave à Ouidah, Bénin " (Ciarcia 2008a). 
d'Abomey sont des représentations non anthropomorphes; à travers la figuration d'animaux et/ou d'objets emblématiques, elles sont censées et illustrer des devises royales, voire des formules légendaires (parfois cryptiques) de la conception du pouvoir et de son rôle, propres à chaque souverain.

Selon la vulgate patrimoniale diffusée lors du lancement de l'itinéraire, les six «stations» ont été conçues pour représenter les séquences du "calvaire» des esclaves : après la vente sur la place aux Enchères, leur transit autour de l'Arbre de l'Oubli - neuf fois pour les hommes, sept fois pour les femmes - aurait constitué une sorte de passage rituel permettant l'effacement de tous les indices de leur appartenance. Ce rituel aurait eu comme contrepoint, contradictoire à vrai dire, leurs circonvolutions (trois fois) autour de l'Arbre du Retour qui auraient permis à leurs âmes, après la mort, de revenir sur la terre des origines. Au lieu dit Zomä̈, "là où le feu ne rentre pas", deux statues qui représentent un homme et une femme agenouillés, ligotés et muselés rappellent aux visiteurs le marquage au fer rouge et la réclusion des esclaves dans les cases où ils attendaient leur déportation. Adjacent à cette installation, le mémorial de Zoungbodji est édifié sur l'emplacement prétendu de la fosse commune où les esclaves décédés auraient été enterrés. Au terme de cet itinéraire, la Porte du Non-Retour, faisant face à la mer sur la plage, est le monument éminent qui a été inauguré, lors de la journée internationale de la Tolérance, le 30 novembre 1995, par le président de la République du Bénin de l'époque, Nicéphore Soglo, ainsi que par le secrétaire général de l'ONU, Boutros Boutros-Ghali, et par le directeur général de l'Unesco, Federico Mayor.

La réalité historique et les interprétations

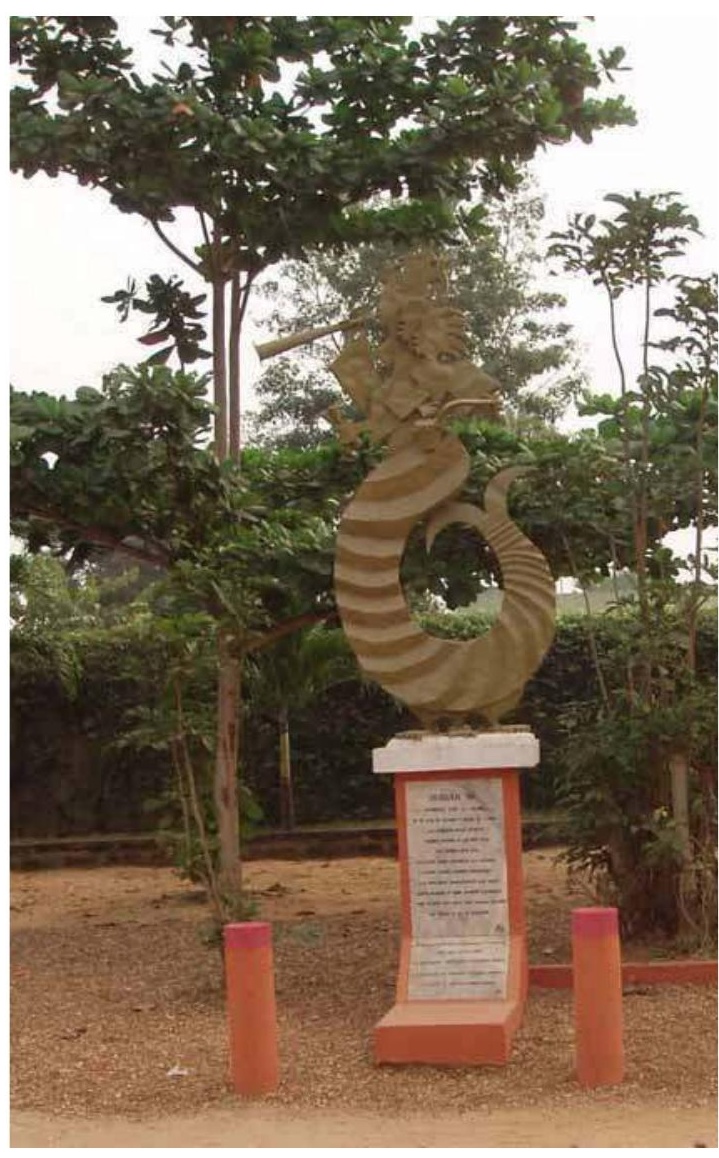

Site de l'Arbre de l'Oubli, Route de l'Esclave, Ouidah, Bénin. Fuvre de Dominique Gnonnou "Kouas" (cl. Gaetano Ciarcia, 20। I). actuelles de ces étapes ne sont pas attestées par les sources documentaires. Robin Law (2004), spécialiste de l'histoire de la région au moment de la traite négrière, s'est appuyé sur un patient travail d'archives pour conclure qu'il n'y aurait jamais eu de place aux Enchères : les esclaves étaient vendus 
à l'entrée des maisons des différents négriers. En ce sens, la place aux Enchères est plutôt la place où se trouvait la maison du négrier de Souza et de ses descendants. La réalité historique d'un Arbre de l'Oubli, d'un Arbre du Retour, d'un lieu de détention et de marquage des esclaves et d'une fosse commune est aussi mise en question par Law, malgré leur élaboration récente dans les récits locaux que j’ai pu récolter à plusieurs reprises. D'ailleurs, toute reconstruction contemporaine se voulant véridique est confrontée au fait qu'à l'époque de la traite, les itinéraires et les modalités du commerce variaient selon les saisons, les périodes, les destinations des captifs, l'efficacité des actions militaires menées par la marine anglaise après l'abolition de la traite en 1807.

En 2005, à la suite de mes premières enquêtes, j'avais émis l'hypothèse que, sur la Route de l'Esclave, certains sites intégrant l'histoire orale de la ville avaient été transformés en lieux de mémoire correspondant aux usages actuels du passé de la traite. Par exemple, l'arbre autour duquel, d'après les chroniques du temps, se déroulaient des négociations entre les négriers européens et les autorités dahoméennes est devenu l' "Arbre de l'Oubli»; l'emplacement de l'arbre où Agadja, le premier roi aboméen de Ouidah, après sa conquête de la ville, en 1727, aurait goûté pour la première fois du gin européen, est devenu le site de l'«Arbre du Retour». Pour ce qui concerne l'actuel "Arbre de l'Oubli", d'autres narrations et documents d'archives font état d'un "Arbre du capitaine » ou "des capitaines " ${ }^{5}$ près duquel, au XVIII siècle, tout nouveau directeur du fort français de Ouidah, lors de son arrivée, était reçu par le Yovogan (littéralement en fon : "chef des Blancs ", c'est-à-dire le dignitaire qui représentait le roi d'Abomey auprès des Européens). À ce propos, il est intéressant de signaler que, comme le remarque aussi Law à partir de ses sources, lors de cette cérémonie, trois circonvolutions autour de l'arbre auraient effectivement eu lieu :

"Le nouveau directeur [du fort St-Louis, dit aussi "fort français”] débarque à Juda [...]. Sur la grève l'attend une très simple chaise de porteur [...]. Le chef du poste côtier, prévenu dès l'arrivée du navire par des actifs guetteurs, se porte à la rencontre du Blanc afin de lui offrir en guise de bienvenue une calebasse emplie d'eau fraîche; il reçoit en échange de l'eau-de-vie et, satisfait, conduit notre directeur sous l'“Arbre du capitaine" où Yavogan [sic] ne tarde pas à les rejoindre: le gouverneur noir paraît dans toute sa pompe, vêtu de soies éclatantes, abrité sous un large parasol, et précédé d'un cortège bruyant et gambadant qui fait trois fois le tour de l'arbre; il présente à son tour au directeur un verre d'eau, un verre de vin et un verre d'eau-de-vie, lui demande aimablement des nouvelles du roi et de la reine de France " (Berbain 1942: 61-62).

5. Cf. Robin Law (2008) qui remarque également, comme j’ai pu faire le constat au cours de mes enquêtes, que les deux Arbres aussi sont parfois nommés par la même épithète Agadjatin (l'«Arbre d'Agadja »). 
Aujourd'hui, la Route de l'Esclave se présente dans un état d'abandon dû en partie à l'incurie des institutions; cette négligence est aussi le reflet d'un manque d'intérêt réel et de financements qui, après la fin du mandat du président Soglo, a caractérisé les politiques nationales de valorisation du patrimoine. D'ailleurs, les espoirs d'un développement d'un tourisme culturel au début des années 1990 se sont envolés par manque d'investissement conséquent de la part de l'Unesco et de l'État ${ }^{6}$. En 2001, le Centre du patrimoine mondial a rejeté une demande de classement du site sur la liste des biens culturels de l'humanité pour défaut de visibilité et de lisibilité d'espaces qui apparaissent encombrés par des éléments architecturaux ne respectant pas l' "esprit des lieux ${ }^{7}$. Cependant, comme je le remarquais déjà il y a quelques années, la situation n’est pas figée. La Route de l'Esclave est devenue l'objet d'affrontements plus ou moins larvés entre plusieurs entrepreneurs privés, issus de la "société civile» qui, profitant d'une juridiction défaillante en matière de protection du patrimoine national, ont occupé des terrains se trouvant sur l'itinéraire officiel ou lui étant adjacents. Leur but était la promotion d'activités commerciales qui supposaient également une forme d'investissement dans la revitalisation de la mémoire, parfois en contradiction avec le projet initial, qui, lui aussi, était hétéroclite. Ainsi d'autres lieux de mémoire en viennent-ils à s'ajouter en quelque sorte à ceux issus de la tentative déjà désordonnée de donner au parcours une cohérence mémorielle et symbolique. Le bâtiment encore en construction, Zomachi, "là où la flamme ne s'éteint pas ", par exemple, édifié derrière le lieu dit Zomä̈, "là où le feu ne rentre pas", représente une sorte de réponse morale, se voulant éclairante et optimiste, à Zomaï, espace des ténèbres et de la résignation. Les récits locaux présentent plusieurs versions de cette traduction; l'image $\mathrm{du}$ "feu " peut être remplacée par celle de la "flamme» ou de la "lumière ". Loin d'être anodines, ces variations semblent exprimer l'attribution de diverses significations, métaphoriques ou symboliques, au prétendu lieu d'enfermement des esclaves. Si le feu est lié au danger d'incendie, la lumière s'oppose à l'obscurité dans laquelle les esclaves étaient gardés, mais elle évoque aussi les vertus de l'espérance et de la raison; la flamme rappelle le feu, mais

6. Actuellement, un projet de développement de grande envergure est destiné à réaménager l'aire géographique située entre la capitale économique Cotonou et les environs de Ouidah. Ce Projet d'aménagement touristique sur la "Route des Pêches" mise sur une croissance massive d'un tourisme de mémoire ayant, parmi ses attractions, la beauté d'un paysage lacustre et côtier encore relativement préservé. Pourtant, le chantier de réalisation, qui va intéresser les villages de la côte, semble avancer d'une manière autonome par rapport à l'action plus proprement culturelle menée par l'État béninois et par d'autres institutions officielles sur la Route de l'Esclave, qui croise la Route des Pêches à la hauteur de la Porte du Non-Retour.

7. Cf. Division du Patrimoine culturel [s. d.]. 
aussi la mémoire et le souvenir. À la différence de Zomä̈, Zomachi n'entend pas commémorer l'obscurité comme effacement de l'identité, mais la lumière toujours vivante, et par conséquent la reconnaissance des origines déchirées. Cette construction est destinée également à devenir à la fois un hôtel (L'Escale du Retour) et un centre de "conscientisation, de repentir et de retrouvailles avec la diaspora douloureuse ", d'après l'inscription lisible sur un panneau situé à l'entrée. Le concepteur et propriétaire, le sociologue Honorat Aguessy, fondateur à Ouidah de l'Institut de développement des échanges endogènes, est aussi le promoteur, depuis 1998, d'une Marche du repentir, devenue par la suite la Marche du devoir de mémoire et du repentir, qui se déroule le troisième dimanche de janvier à partir de la place aux Enchères. Grâce à cette cérémonie, qui invite à dépasser les divisions et les blessures du passé de l'esclavage, la société civile béninoise devrait être en mesure d'assumer une partie de la « responsabilité morale " de cette histoire.

L'autre élément qui rend encore plus laborieuses la lecture et la visibilité patrimoniale du site est représenté par l'action de l'ONG ProMeTra (Promotion de la médecine traditionnelle), dont l'activité principale consiste à rechercher et à diffuser des méthodes et remèdes thérapeutiques empruntés à la pharmacopée populaire. Implantée dans une dizaine de pays, parmi lesquels la France et les États-Unis, ProMeTra a fait construire, sur la plage, à quelques centaines de mètres de la Porte du Non-Retour, la Porte du Retour avec son musée de la Diaspora. Là aussi, nous pouvons observer une entreprise commerciale visant à valoriser la mémoire de la traite, et s'adressant prioritairement aux visiteurs d'origine afro-américaine sensibles au thème du retour à la « Mère Afrique ». Dans ce cas de figure, les idées clés ne sont ni le repentir ni la réconciliation, mais les "retrouvailles " avec une Afrique mythique, mise en scène à travers des représentations de cérémonies vodun. D’après les promoteurs de cette opération, Ouidah devient à la fois la destination d'un retour momentané de " pèlerins " afroaméricains, associés à des visiteurs européens et américains n’ayant aucune origine africaine, et le point de départ pour une "connexion spirituelle » avec le vodun réinterprété comme religion ancestrale et matrice commune d'un œecuménisme aux fonctions cathartiques de type new age.

\section{Généalogies antagonistes et cheminements polémiques}

Après le refus de la demande d'inscription sur la Liste du patrimoine mondial de l'Unesco par le Centre du patrimoine mondial, un nouveau dossier a été préparé par un comité d'experts béninois. De ce document dont, au cours du mois de janvier 2011, j'ai pu consulter une version 
provisoire (mais, selon mes interlocuteurs impliqués dans le projet, presque définitive), de nouveaux lieux de mémoire du passé de l'esclavage présents sur le territoire béninois intègrent l'itinéraire de la Route de l'Esclave. Il s'agit des sites archéologiques d'Oké Shabé - près de la ville de Savè, située dans la région des Collines à environ cent cinquante kilomètres d'Abomey - et de Yaka, dans la commune de Dassa-Zoumé toujours dans la région des Collines -, où il est possible de retrouver les vestiges d'emplacements défensifs (grottes, remparts, abris, points d'observation, tours de contrôle) habités et utilisés par des groupes d'origines différentes, tous affectés par des guerres et des razzias ayant eu pour conséquence la mise en esclavage des populations vaincues.

En ce qui concerne l'itinéraire traversant la commune de Ouidah, deux autres sites ont été retenus : la forêt sacrée de Yênouzun, lieu rituel de transit des convois de captifs, dans l'arrondissement périphérique de Savi - ancienne capitale du royaume huéda, vaincu par les Fon d'Abomey en 1727, située à environ huit kilomètres de Ouidah -, et l'ancien fort portugais dans le centre de la ville, devenu par la suite le musée Historique, qui, durant la période de la traite, aurait servi d'entrepôt et de forteresse. Pour ce qui relève des étapes déjà sélectionnées comme significatives dans l'ancien dossier, le Mémorial de Zoungbodji est désormais présenté comme une sorte de mausolée du site archéologique nommé la « fosse aux esclaves ».

Par ailleurs, au lieu de mettre en valeur la Porte du Non-Retour considérée comme trop métaphorique, le nouveau projet privilégie l'inscription patrimoniale de l'espace adjacent dit "L'embarcadère de Djègbadji ». Ce site est considéré comme étant plus fidèle à une reconstitution des lieux de mémoire de la déportation des esclaves. Ainsi la Porte du Non-Retour estelle destinée à devenir le monument emblématique conférant une dimension mémorielle tangible au prétendu « lieu véridique » de l'embarquement des cargaisons d'esclaves dont il ne reste plus de traces physiques. Un jeu scénographique de combinaisons, entre les lacunes de l'archéologie et les récits de commémoration, semble donc accompagner la volonté de donner une plausibilité historique à l'itinéraire afin qu'il puisse être reconnu comme un bien culturel protégé par l'Unesco. D'une manière implicite, une telle invention nous renvoie à ce que James E. Young considère comme «le syndrome de la tombe absente " $(1993: 729 \text { sq. })^{8}$, se manifestant, par exemple, dans des sites où l'impératif de rendre compte de la Shoah ne peut être que confronté à celui de rendre visible la disparition.

Ainsi que tous mes interlocuteurs impliqués dans la conception de ce nouveau programme de valorisation de la Route de l'Esclave me l'ont

8. Dans cet article, Young reprend le travail de Joost Meerloo (1968). 
signalé, il s'agit, du point de vue de sa lisibilité patrimoniale, d'un « dossier

à problèmes ». Les principaux points considérés comme sensibles de la part des experts in loco et mandatés par l'Unesco sont les suivants :

- La question de l'authenticité des sites. Les membres du comité national de spécialistes ont remarqué qu'il était impossible d'authentifier certains lieux. En conséquence, d'après les nouvelles directives, le principe d'exhaustivité doit céder la place à une logique sélective prenant en compte seulement des éléments à la valeur avérée et "nécessaire". Parmi les sites réputés tels et dont la réalité historique est sûrement ou relativement prouvée : les sites d'Oké-Shabé et Yaka dans la région des Collines, de la Forêt sacrée de Yênouzun à Savi, de la Place aux Enchères, de l'Arbre de l'Oubli, de la fosse commune de Zoungbodji, de l'Arbre du Retour, de l'embarcadère de Djégbadji, à côté de la Porte du Non-Retour.

- L'«altération" architecturale de certains espaces comme la place aux Enchères, envahie par la maison familiale des de Souza, qui ont d'ailleurs rebaptisé l'endroit "Place Chacha " du surnom du fondateur Francisco Felix de Souza, devenu après sa mort le titre honorifique désignant le chef du lignage de Souza.

- Le bâtiment imposant et toujours en construction de Zomachi, qui encombre le parcours en en occupant physiquement une partie, et en détournant du point de vue interprétatif la signification mémorielle qui lui avait été attribuée par les auteurs du projet de la Route de l'Esclave ; il s'agit d'un espace qui aurait dû normalement faire l'objet d'une expropriation patrimoniale de la part de l'État béninois.

- Les statues représentant les rois aboméens qui sillonnent le trajet et interdisent, d'après certains responsables du patrimoine, une lecture éminemment anti-esclavagiste et acceptable - à la fois pour les membres de la «diaspora» et pour les descendants des populations ayant été victimes du pouvoir dahoméen - du passé de l'esclavage dans la région.

Ces "problèmes " qui empêchent une reconnaissance internationale officielle (au niveau de l'Unesco) de la Route de l'Esclave en tant que bien patrimonial ayant un intérêt culturel indiscutable, sont la conséquence d'une histoire complexe, à la fois sociale et politique, ancienne et très récente. Le cas des sculptures figurant les rois d'Abomey semble être, à ce titre, particulièrement révélateur.

D’après Nouréini Tidjani-Serpos, intellectuel béninois, professeur d'université et ancien vice-directeur de l'Unesco, avec lequel je me suis entretenu à plusieurs reprises, la situation actuelle de la Route de l'Esclave serait le prolongement d'une relation paradoxale de concurrence symbolique avec une manifestation qui a précédé et - pourrait-on dire - préparé son lancement: le Festival d'arts et de la culture vodun, "Ouidah 92. 


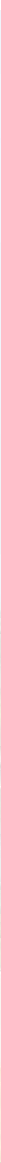

Entrée de la Porte du Retour, site créé par l'ONG ProMeTra (Promotion de la médecine traditionnelle), Ouidah, Bénin. Les statues représentent la "Mère Afrique" accueillant les "pèlerins" de la diaspora afro-américaine. Euvre de Benjamin Mafort (cl. Gaetano Ciarcia, 20 I I). 


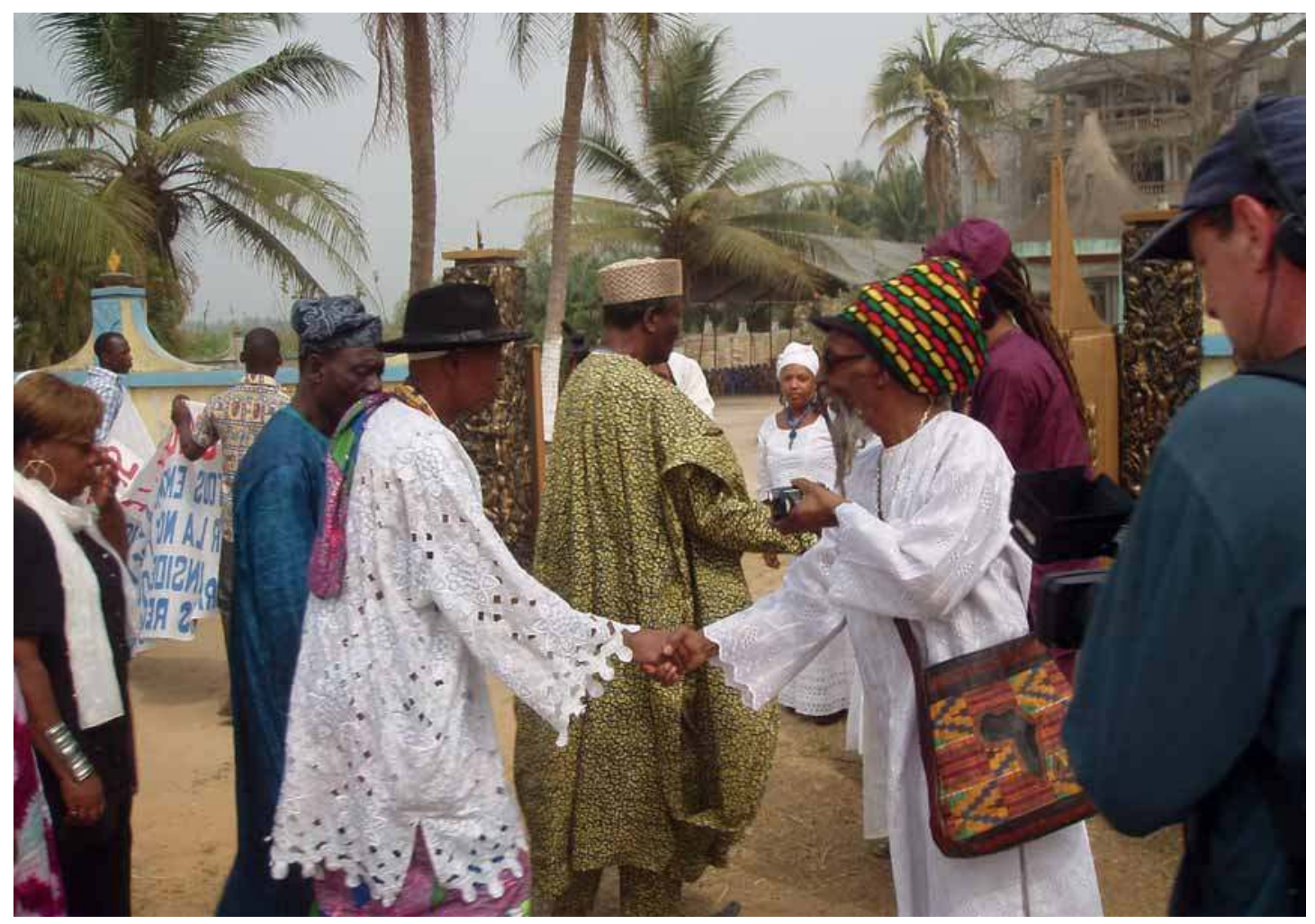

Rencontre des participants à la Marche du devoir de mémoire et du repentir avec un groupe d'Afro-Américains.

Site dit Zomachi, Ouidah, Bénin (cl. Gaetano Ciarcia, 20l2). 


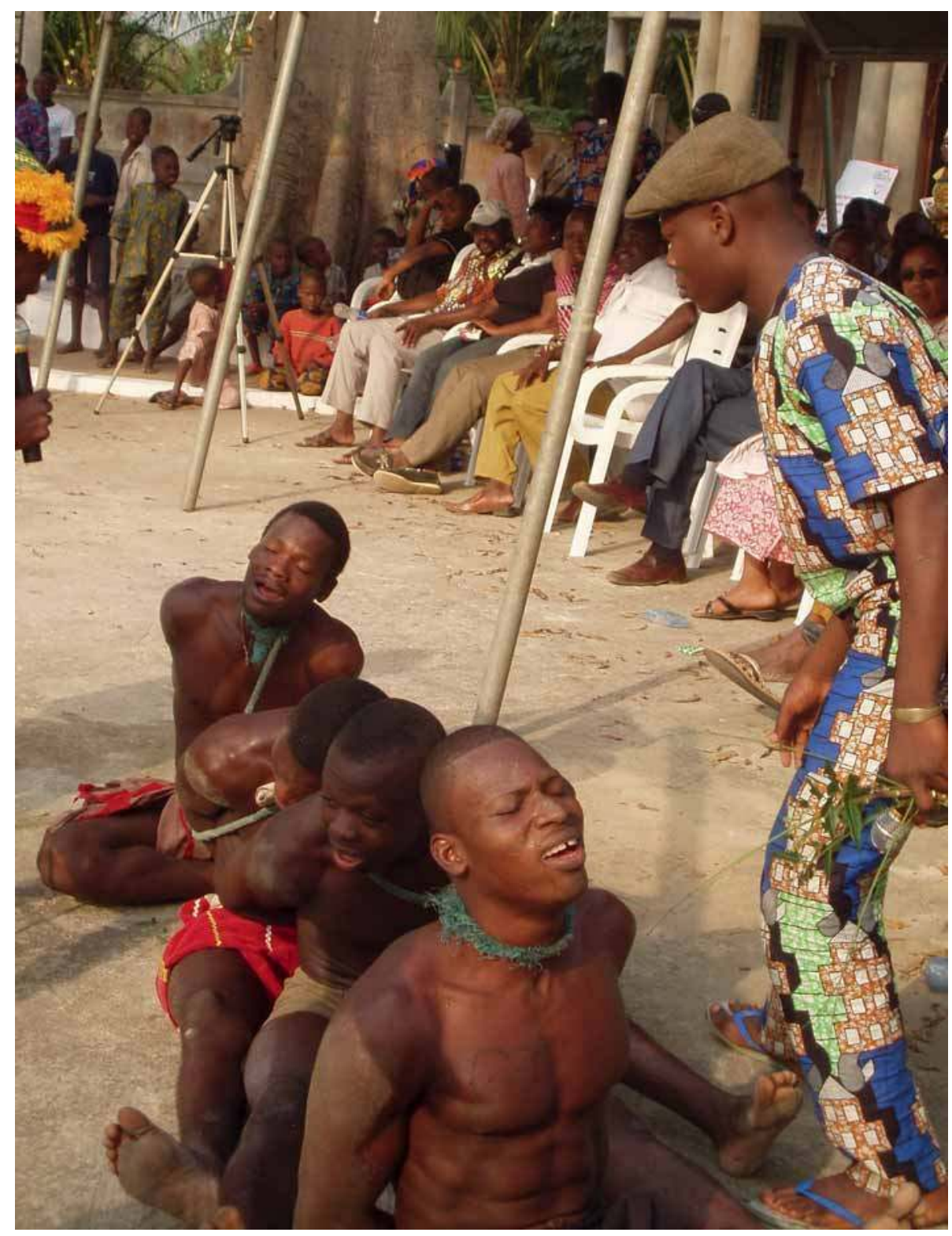

Représentation d'esclaves

Cérémonie de clôture de la Marche du devoir de mémoire et du repentir. Site dit Zomachi, Ouidah, Bénin (cl. Gaetano Ciarcia, 201 I). 


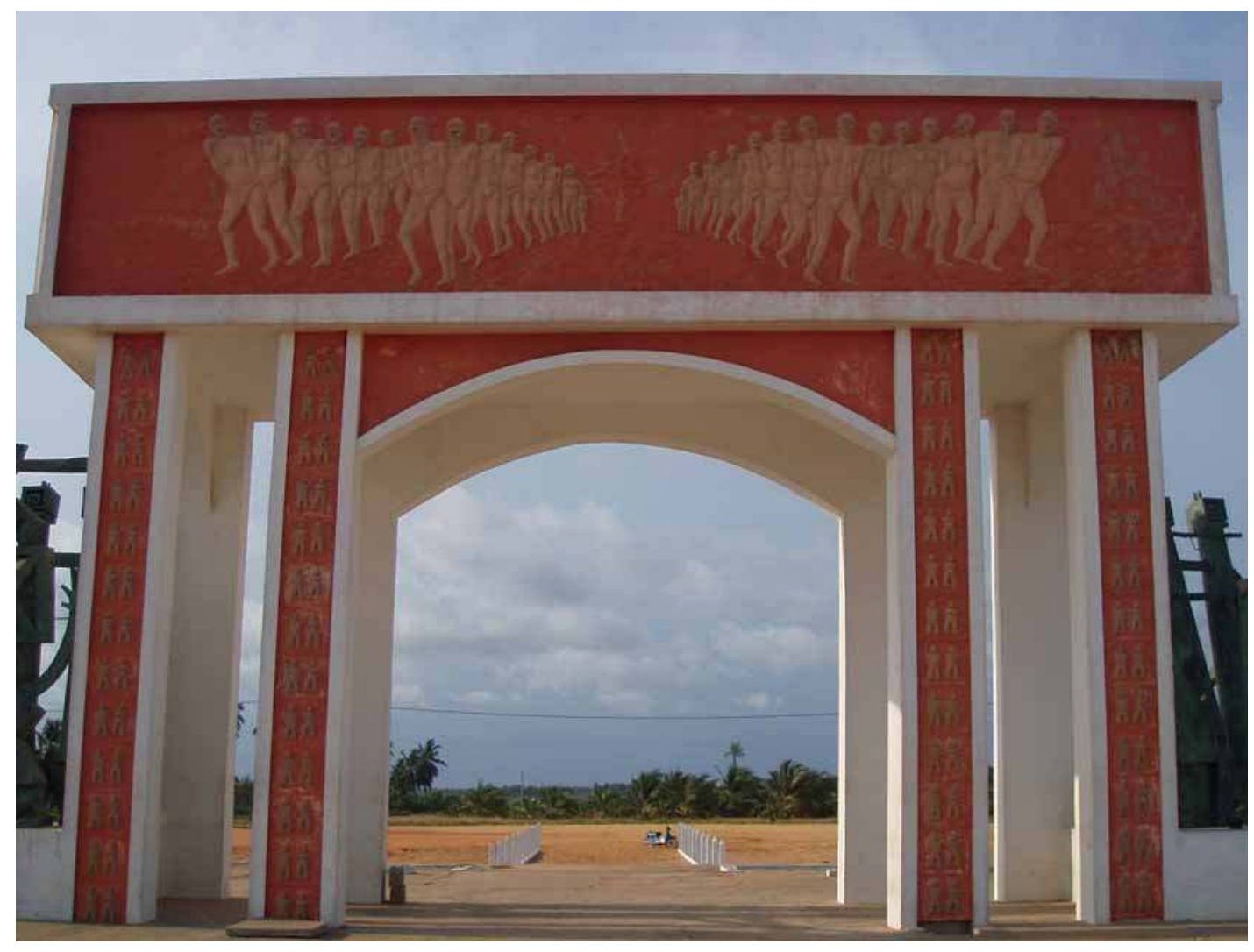

\section{Porte du Non-Retour}

Route de I'Esclave, Ouidah, Bénin. Fuvre de Fortuné Bandeira

(cl. Gaetano Ciarcia, 2005). 
Retrouvailles Amériques-Afrique », en février 1993. Tidjani-Serpos a été le concepteur de ces deux moments fondateurs du renouveau des cultes vodun au Bénin et de leur connexion avec la commémoration des mémoires de l'esclavage. Selon son récit, le projet de la Route de l'Esclave avait été soutenu au sein de l'Unesco par deux pays : Haïti et le Bénin. Pour des questions d'enjeu politique, il fallait trouver un argument convaincant pour soutenir la candidature du Bénin comme pays susceptible d'accueillir une mémoire emblématique de l'époque esclavagiste et de susciter des rapprochements au sein de la diaspora noire. Car, du point de vue démographique, la diaspora francophone, fortement minoritaire par rapport aux diasporas anglophone et lusophone, desservait l'aspiration de l'État béninois à accueillir le centre mémorial d'un tel programme. De sorte que, d'après une communication personnelle de Tidjani-Serpos, c'était le "religieux ", donc le vodun, qui était en mesure d'être investi par les actions et rhétoriques patrimoniales, et de devenir le «liant» culturel et historique justifiant le choix de son pays comme foyer du projet international de l'itinéraire. La difficulté de «faire passer " le Bénin a été aussi surmontée par l'organisation de Ouidah 92 en tant qu'épicentre artistique et culturel apte à créer les conditions de circulation d'une vulgate légitimant une nouvelle ère d'échanges entre les communautés afro-américaines et les institutions internationales comme l'Unesco.

Toujours selon Tidjani-Serpos, dans les années 1990, la constitution d'un patrimoine d'œuvres d'art intégrant la nouvelle fondation de l'État qui sortait d'une période de dictature, et qui, à l'époque, était engagé dans un processus de "renouveau démocratique ", avait été un des objectifs à l'origine de Ouidah 92. Afin d'encourager leur créativité, les artistes béninois avaient été incités à "plonger dans le vodun [de façon] à restituer leurs expériences initiatiques à travers leurs représentations visuelles ». En ce sens, l'institution d'une culture nationale était envisagée comme élaboration in fieri d'une relation et d'une perception d'un vécu cultuel et esthétique de l'expérience religieuse traditionnelle dont la sphère du vodun devenait le réceptacle authentique. Les artistes sollicités et choisis étaient donc perçus comme des émissaires en provenance des mondes sociaux de la coutume. Ils devaient inventer des figures esthétiques exprimant une continuité entre les pratiques traditionnelles et les mutations modernes, et, par conséquent, souligner la relation que la société béninoise contemporaine devait entretenir avec ses cultes toujours pratiqués par une partie de la population, tout autant que représenter l'esclavage qui s'inscrit de fait dans son passé précolonial.

Dans ce cadre, comme Tidjani-Serpos me l'a dit, "Ouidah 92 est Ouidah 92 ", c'est-à-dire l'organisation d'un festival n'ayant pas encore en 
vue l'inscription de lieux sur la liste de l'Unesco : les statues commémorant les rois d'Abomey demeuraient des représentations d'une histoire nationale en chantier qui n'avait pas (encore ?) pour objectif leur projection et reconnaissance sur la scène patrimoniale de la traite négrière, telles qu'elles seront évoquées quelques années plus tard grâce à l'aménagement de la Route de l'Esclave. Une telle explication suppose qu'une superposition et une conjonction de motifs mémoriels soient mises en place, mais qui, si elles sont le fait du même initiateur, Tidjani-Serpos, relèvent d'un programme général utopique, plein de contradictions et d'anachronismes. Du point de vue d'une économie morale de la transmission du passé de l'esclavage, cette version de l'histoire récente de l'héritage culturel montre les difficultés qui surgissent quand, au projet de constitution d'une dynastie "nationale» - celle représentée par la monarchie aboméenne -, s'ajoute la délimitation d'un territoire consacré aux usages mémoriels et susceptible de dégager des significations "diasporiques». Comme l'a remarqué Paul Ricœur à propos du travail d'Henry Rousso sur la mémoire de Vichy où le souvenir de la Collaboration s'oppose de facto à celui de la Résistance, "voir une chose, ce n'est pas en voir une autre. Raconter un drame, c'est en oublier un autre" (2000 : 584). Dans le cas de la Route de l'Esclave, c'est plutôt l'affrontement, dans un même lieu (et en même temps), de deux perspectives de toute évidence antagonistes : pour l'une, donner à voir le prestige et la puissance des souverains du Danhomé supposés préfigurer l'État moderne béninois et, pour l'autre, faire entendre la souffrance que la même dynastie, compromise dans la mise en esclavage locale et dans la traite transatlantique, a infligée aux populations qu'elle avait assujetties. En ce sens, sur la Route de l'Esclave, examinée à travers le prisme de Ouidah 92, nous pouvons observer des tentatives diverses, conflictuelles, voire contradictoires de " transformer en histoire " (Tchitchi 2007 : 92) l'injonction patrimoniale du souvenir et la généalogie d'une construction rhétorique récente, entre autres matérialisée par les œuvres des artistes. La restitution esthétique et cultuelle du passé de l'esclavage va ici de pair avec des pratiques politiques mémorielles d' " organisation de l'oubli » (Ricœur 2000 : 582). Il s'agit, en effet, d'un processus de réinterprétation du passé qui s'actualise sans cesse à travers les conjonctures politiques. $\mathrm{Si}$, «en créant des espaces communs de la mémoire, les monuments propagent l'illusion d'une mémoire commune» (Young 1993: 735), la fabrication de ce genre de récit, comme l'auteur de cette réflexion l'observe pertinemment, tente de situer les événements du passé en invoquant une "séquence linéaire " que le monument est censé inscrire dans un «ordre cognitif». Sur la Route de l'Esclave, une telle démarche provoque des discontinuités dans la perception historique et les significations 
topographiques du paysage mémoriel en chantier. En ce sens, il nous semble toujours d'actualité d'interroger l'amnésie et sa genèse, dont le monument, sur la scène publique de la remémoration, est respectivement le témoin et le gardien :

"C'est justement parce que les monuments semblent se souvenir de tout sauf de leur propre passé, que notre fonction critique pourrait être de réinvestir le monument de sa mémoire de soi, de sa propre construction, de ses origines " (Ibid. : 736).

Origines que le présent concrétise et que, si l'on décline la célèbre leçon lévi-straussienne, le monument, tel un masque, transforme sans cesse (Lévi-Strauss 1975).

Devenues emblématiques de la domination qu'autrefois le Danhomé avait exercée sur le reste du territoire national d'aujourd'hui, et ce, depuis leur élévation dans la première moitié des années 1990, à l'époque de la présidence de Nicéphore Soglo, lui-même d'origine aboméenne, les statues ont constitué un enjeu politique et suscité une polémique quant à la question mémorielle et artefactuelle. Lors de la dernière campagne électorale en 2011, au moment où se dessinait une alliance électorale "méridionale » contre le président de la République sortant Boni Yayi, identifié comme "nordiste», réélu néanmoins, cette question était redevenue particulièrement sensible du fait même que les statues étaient à ce moment-là inamovibles.

\section{Le retour, figure rituelle et patrimoniale de l'oubli}

Comme le montrent les descriptions précédentes, la relation entre les Arbres de l' "oubli » et du «retour» joue un rôle capital dans le récit de l'épopée tragique vécue par les captifs juste avant leur déportation vers les Amériques. Ce récit, Tidjani-Serpos se revendique en être aussi l'auteur: "C'est ma prose!", s'est-il exclamé au cours de l'un de nos entretiens?. Il faisait ainsi allusion aux plaques qui décrivent et interprètent les diverses étapes de l'itinéraire, parmi lesquelles celles mettant au jour l'existence, lors de la déportation des convois d'esclaves, d'une sorte de rite de passage imposant aux captifs l'oubli de leur identité et d'une liturgie de la promesse qui leur aurait été faite d'un retour de leurs «âmes » sur le sol ancestral après leur mort.

9. Parmi les sources locales de sa narration, Tidjani-Serpos cite feu daagbo Hunon Huna et les membres âgés de l'UGDO (Union générale pour le développement de Ouidah), une association composée, depuis le milieu des années 1980, par un groupe de notables et d'intellectuels qui se sont constitués en groupe de pression politique et économique. En misant entre autres sur la richesse du passé historique et du patrimoine immobilier de leur ville, l'action de l'UGDO comptait sur l'apport potentiel de la coopération internationale et du tourisme. Sur le rôle joué par l'UGDO dans la valorisation culturelle du passé de l'esclavage et de sa connexion avec le renouveau culturel du vodun, cf. UGDO (1985). 
Dans son essai Les Formes de l'oubli (2001), Marc Augé analyse le retour comme une des trois figures de l'oubli à l'œuvre dans les rituels africains. Dans les pratiques de la possession, le principe du retour serait animé par «l'ambition première [...] de retrouver un passé perdu en oubliant le présent - et le passé immédiat avec lequel il tend à se confondre - pour rétablir une continuité avec le passé plus ancien, éliminer le passé "composé" au profit d'un passé "simple" " ${ }^{10}$. L'oubli rendrait donc possible de penser la continuité, là où cette continuité n'est que l'effet de l'institution cérémonielle et/ou commémorative d'une paradoxale épiphanie momentanée du temps qui fut. À ce sujet, Marc Augé nous dit :

«Rare, éphémère, instable, la figure du retour est heureusement réversible. Très significativement, les individus et les cultures, prenant acte des difficultés intrinsèques à l'acte de revenir, l'imaginent accompli " (Ibid. : 103).

Ces remarques peuvent introduire et peut-être partiellement répondre à l'intérêt que me paraît susciter un examen approfondi de la relation problématique entre les motifs mémoriels à l'œuvre sur la Route de l'Esclave. Lors de mes premières enquêtes au Bénin, j’ai perçu d'emblée ces motifs comme incongrus du point de vue d'une véritable reconstitution historique, et, dans le même temps, j'ai pu constater leur intégration diffuse dans les narrations locales sur le passé de l'esclavage mis en scène et raconté par la Route dans son espace iconique et épigraphique. Matérialisés et représentés par la présence des deux Arbres, ces deux thèmes "de l'oubli » et " $\mathrm{du}$ retour» sont censés être les traces de l'unité topographique et temporelle dudit parcours et de son récit. Elles participent ainsi de l'institution d'un héritage historique qui semble refléter la nécessité rituelle d'un agencement entre oubli et retour, comme elle a été observée par Marc Augé.

À partir de ces hypothèses, je vais tenter d'analyser le discours officiel et les récits locaux qui l'ont alimenté et qui continuent de le réinterpréter sans cesse comme un mythe. Que, depuis deux décennies, sur la Route de l'Esclave, il y ait eu une articulation entre une tradition iconographique en gestation, des narrations populaires et des rhétoriques patrimoniales, nous semble pouvoir relever d'une approche anthropologique soucieuse de prendre en compte l'interaction entre les figures narratives orales, écrites, rituelles et visuelles des mémoires officielles et des mémoires locales de l'esclavage dans le Bénin méridional, mais également à l'échelle prétendument diasporique et globalisée ${ }^{11}$. En s'appuyant sur l'analyse de

10. Les deux autres figures ou formes de l'oubli perçues par Marc Augé dans certains rituels africains sont celles du suspens et du commencement (ou du re-commencement).

11. Sur ce sujet, j’ai trouvé matière à réflexion dans le livre de Carlo Severi (2007). 
Carlo Severi, il est possible de penser la «mémoire montrée » (2007: 260) sur la Route de l'Esclave comme anthropologiquement significative du caractère réflexif de l'action commémorative patrimoniale en tant que lieu suscitant l'adhésion à des narrations et donc au fonctionnement de croyances. Cet effet performatif peut aussi se repérer dans «l'acquisition rituelle d'une identité complexe, à travers une série cumulative de connotations contradictoires» (Ibid. : 287) et provoquer des illusions mémorielles ${ }^{12}$. Par rapport à ce cadre interprétatif, il est nécessaire de rappeler que, dans le contexte en question, la représentation hétéroclite du passé est également une conséquence de l'injonction adressée, lors de Ouidah 92, aux artistes béninois de connecter leur art, se voulant aussi un héritage historique à venir, avec l'imaginaire émanant de leurs connaissances ou pratiques du vodun. Dans le cas des Arbres, les deux artistes béninois chargés de donner à voir, du point de vue plastique, les notions de l'oubli et du retour ont suivi cette recommandation. D'ailleurs, ce genre de travail de réélaboration esthétique d'entités cultuelles, en quelque sorte "re-sémantisées », est à l'œuvre d'une manière diffuse dans le contexte contemporain d'institution mémorielle du passé de l'esclavage réinterprété à travers les figures médiatrices du sacré traditionnel. En ce sens, dans le Bénin méridional, nous observons l'adéquation créative entre, d'un côté, une morale religieuse acculturée, réclamée comme "endogène", et, de l'autre, les nécessités économiques et les contraintes sociopolitiques actuelles. Par l'attribution de nouvelles interprétations d'images rituelles ou d'entités cultuelles converties en simulacres des mémoires publiques de l'esclavage, une telle configuration donne lieu à l'édification d'une culture patrimoniale ${ }^{13}$. À cet égard, l'œuvre des artistes béninois réinterprétant la "mémoire " exposée de l'oubli et du retour sur la Route de l'Esclave fournit des pistes d'interprétations intéressantes. Dominique Gnonnou Kouas de Porto-Novo est l'auteur de la statue de Mami Wata (déesse de la mer représentative d'un imaginaire vodun désormais mondialisé), située à côté de l'Arbre de l'Oubli, avec laquelle il a choisi d'évoquer le thème de la perte de l'identité. Pourtant, selon Kouas, la Mami Wata qu'il a forgée joue, avec son

12. Carlo Severi $(2007: 322)$ parle d'«illusion visuelle».

13. Un exemple est donné par l'attribution de significations historiques à des entités cultuelles comme celles représentées par les statues de "revenants» sur l'espace devant la Porte du NonRetour sur la Route de l'Esclave à Ouidah. Comme un dignitaire du culte des egun-gun me l'a fait observer, leur présence sur la plage est aberrante en raison de l'interdit qui frappe leurs "sorties" devant la mer. Cependant, la scénographie muséale à l'œuvre sur le lieu de la Porte du Non-Retour suggère aux visiteurs la transformation des représentations des revenants en simulacres des âmes des esclaves morts de l'autre coté de l'océan, retournant en Afrique, comme leurs descendants devenus des touristes ou des pèlerins de la mémoire. 
clairon, une musique qui empêche l'oubli; en ce sens, elle ne serait donc pas une "sirène qui fait oublier", mais plutôt une sirène qui alerte. Cyprien Tokoudagba d'Abomey est l'auteur de la statue représentant l'Arbre du Retour. D'après son récit, c'est

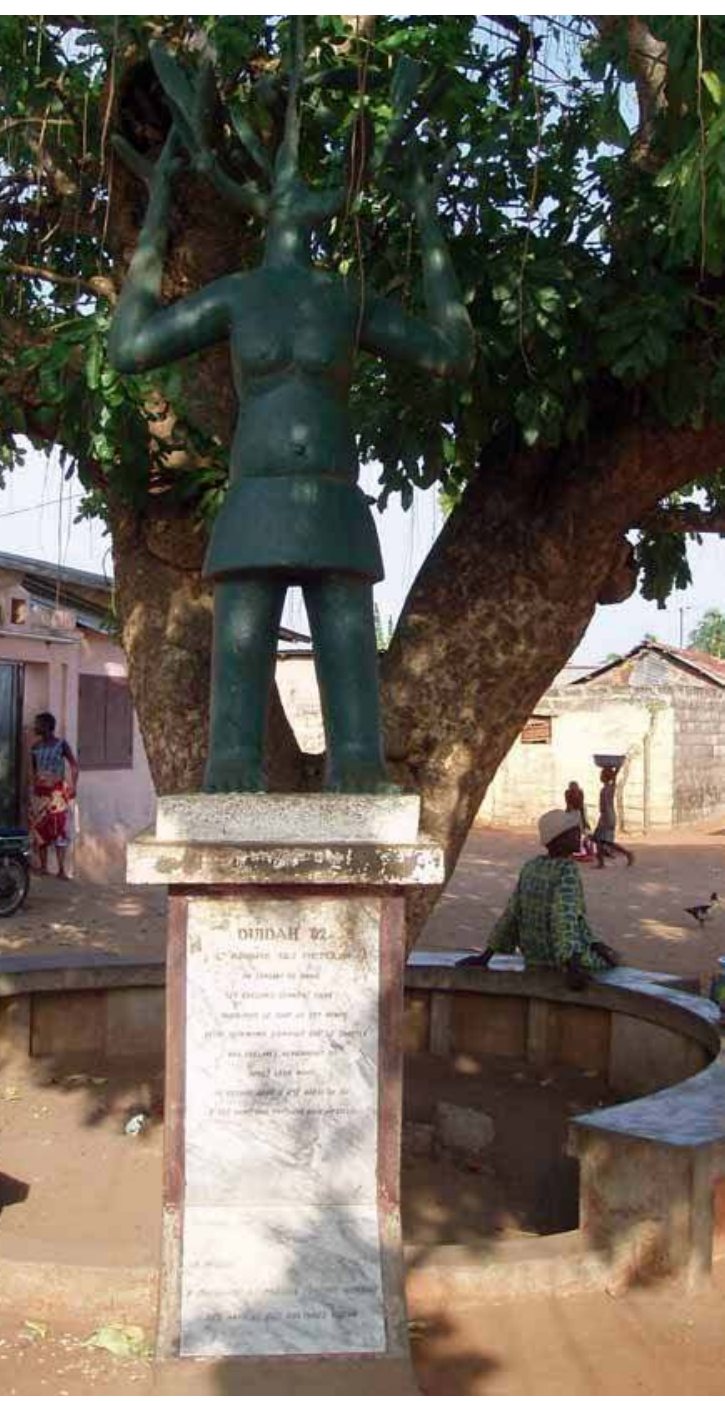

Site de l'Arbre du Retour, Route de l'Esclave, Ouidah, Bénin. Euvre de Cyprien Tokoudagba (cl. Gaetano Ciarcia, 20 I I). après qu'on lui a raconté l'histoire de l'Arbre du Retour, dont il n'avait jamais entendu parler avant qu'il ne reçoive la commande pour Ouidah 92, qu'il s'est mis en quête d'une inspiration pour réaliser son travail. La sculpture côtoyant l'Arbre du Retour qu'il a choisi de créer représente Aziza, la divinité/esprit de la forêt. Aziza est considéré également comme un génie du monde végétal qui peut être appréhendé aussi comme un vodun qui n'a ni fétiches ni " couvents ». Il peut donc être perçu comme un vodun immatériel et sans adeptes; mais, en même temps, il exprime les qualités personnelles de l'individu à qui on reconnaît une efficacité et un domaine bien concrets, chacun étant le propriétaire de son Aziza qui ainsi ne peut faire l'objet d'une spoliation ou d'une dépossession. Selon Suzanne Preston Blier :

«[...] aziza-derived powers, especially talent, skill, good fortune, and inception, constitute the intangibles in each's individuals's life [...] an invisible being that resides deep within the recess of the forest " (1995: 199 sq.).

Il est donc significatif que, pour illustrer le départ et la possibilité d'un retour désincarné, Tokoudagba ait choisi ce vodun qui ne peut pas être enlevé à son détenteur. Si «Aziza est une force qui coiffe beaucoup de choses", selon l'un de mes interlocuteurs béninois, sa dimension polyédrique et la versatilité de ses significations suggèrent que, à l'époque de la traite, l'esprit personnel de l'esclave ne pouvait être supprimé et était donc susceptible de revenir ${ }^{14}$. Toutefois, les Arbres de l'Oubli et du Retour ne mettent pas seulement en scène les

14. Claude Tardits (1958) signale la création, au Dahomey, en 1955, d'un journal dont le titre, Aziza, rappelait, selon ses rédacteurs, le "dieu-guide des hommes égarés». 
étapes d'une épopée cultuelle, mais aussi des notions morales qui fonctionnent comme une sorte de didascalie nécessaire aux motifs de la repentance ou de la culpabilité que nous pouvons envisager comme originairement étrangers à l'ethos des pratiques vodun. En ce sens, si la présence d'une scénographie comportant leur présence est une reconstruction historiquement fictive, son invention relève d'injonctions contemporaines marquées par une pédagogie et par une généalogie de matrice chrétienne (cf. Ciarcia 2008b). Rappelons-le, d'après le dispositif muséal, et à la suite de la vente des esclaves sur la place aux Enchères, leur transit autour de l'Arbre de l'Oubli aurait constitué une sorte de passage rituel permettant l'effacement de tout rappel de leur appartenance. Avant la déportation sur les bateaux négriers, ce rituel aurait eu comme suite et contrepoint leur marche circulaire autour de l'Arbre du Retour qui aurait dû permettre à leurs âmes, après la mort, de revenir sur la terre des ancêtres. Si les sources écrites dont nous disposons ne prouvent pas l'existence, à l'époque de la traite, de ces escales mises en scène aujourd'hui pour les visiteurs de l'itinéraire, selon toute logique, la production d'une amnésie de l'origine et la promesse d'un retour au sol natal scandant les actes de la déportation esclavagiste relèvent d'une incongruité. Pourtant, à Ouidah, comme nous l'avons déjà remarqué, les narrations locales se sont très rapidement appropriées cette interprétation mémorielle. Un tel réinvestissement affectif du passé interroge les modes de transmission à l'œuvre dans les références orales de cette version contradictoire de l'expérience historique vécue par les esclaves. À un niveau plus général, la logique d'un agencement entre l'imposition rituelle de l'oubli et la promesse, elle aussi produite par un passage rituel, d'un retour, déployée sur l'itinéraire promu par l'Unesco, inscrit donc la commémoration publique de la souffrance des esclaves dans une tentative d'anamnèse purificatrice de l'ignominie esclavagiste où l'effort de mémoire pourrait paradoxalement avoir pour issue un oubli libérateur de la tare et du stigmate hérités de la période de la traite négrière. Par exemple, en 2011, à la veille de la Marche du devoir de mémoire et du repentir que, tous les troisièmes dimanches du mois de janvier, Honorat Aguessy, le sociologue concepteur et propriétaire de Zomachi (voir supra), organise, j'avais été intrigué par la lecture d'une banderole de l'IDEE (Institut de développement des échanges endogènes) ${ }^{15}$ : "Que nos sœurs et frères des Amériques et Caraïbes [sic] oublient l'opprobre de l'esclavage».

15. À l'IDEE, chaque année durant son université d'été, Honorat Aguessy développe le concept de "pertinence " qu'au cours de nos entretiens il définissait par la nécessité de "coller à la vie de nos parents par rapport à laquelle les intellectuels sont devenus des étrangers » et " de s'inculturer grâce à l'enseignement des savants et des sachants ». L'endogénie invoquée par Aguessy serait constituée d'une essence primordiale africaine, traumatisée par la traite, la colonisation et la conversion subreptice au monothéisme des missionnaires. L’enseignement des références à une culture.../... 
Qu'il y ait une labilité dans la relation entre aspiration et devoir à se souvenir et droit à l'oubli, cela me semble retentir à travers les positions changeantes du discours. D'ailleurs, une telle indétermination mémorielle est perceptible dans la quête d'une authenticité historique des lieux, celle-ci pourtant difficile à prouver, qui a fini par intégrer la croyance moderne en l'existence d'un "patrimoine immatériel». En ce sens, des reconstitutions architecturales ou des inventions esthétiques sont susceptibles d'assumer un rôle décisif pour la réactualisation de la valeur morale des faits du passé. Au Bénin méridional, l'adhésion répandue à la qualité "immatérielle» de cet héritage m'a semblé être perçue avant tout comme un patrimoine possible, c'est-à-dire réalisant l'inclusion de notions, à la généalogie complexe et incertaine, comme celles d'oubli et de retour, dans une histoire à la fois " commune » (désormais globalisée, mais aussi à vocation nationale) et culturellement originale et originelle. L'immatérialité de ce legs, ou plutôt la conscience vécue de son immatérialité, donnée à voir sur la Route de l'Esclave serait être le résultat d'une distanciation théâtrale sensible au développement patrimonial d'un vodun, vecteur d'une morale traditionnelle et de sa connexion thématique contemporaine avec les mémoires de l'esclavage.

Au cours de mes enquêtes, les réponses concernant la conjonction entre figures de l'oubli et du retour validaient généralement l'idée que les effets violents de la souffrance infligée aux esclaves nécessitaient d'être maîtrisés à travers des techniques de fabrication rituelle d'une sorte d'amnésie. Toutefois, cette perte de soi n'empêchait pas que la malédiction émanant de leur colère pût coexister avec des sentiments de "compassion » ou de crainte des négriers qui, avant l'embarquement des captifs, auraient préparé le retour spirituel de leurs âmes. Les récits que j'ai recueillis à Ouidah et à Abomey s'accordent à dire que les convois provenant d'Abomey étaient constitués d'esclaves ligotés mais « en furie». Les rotations autour de l'Arbre de l'Oubli pourraient alors se comprendre non seulement comme un rituel d'effacement de l'identité, mais aussi comme une technique d'incantation et d'exténuation de la douleur des captifs. Pour ce qui concerne l'Arbre du Retour, un de mes interlocuteurs y voyait l'expression du souhait de dédouaner les organisateurs de leur déportation de vouloir intentionnellement l'opérer. Selon ses dires, si la violence perpétrée était un signe ou la manifestation de la puissance des dominants,

[Suite de la note 15] perdue et à retrouver est donc confié à des chercheurs universitaires (les "savants ») et à des personnes-ressource définies comme des " sachants » : thérapeutes traditionnels, dignitaires, chefs de culte, rois, devins, conteurs. Au sein de cette fondation en cours d'une culture " animiste", traditionnelle et moderne, écrite et orale, les traces et les détours de la mythologie en chantier intègrent l'élaboration de diverses pratiques commémoratives de la traite négrière. 
les sbires de ces derniers savaient aussi que cette violence aurait pu avoir des "conséquences" néfastes, procédant d'une vengeance posthume des esclaves. Ne comportant pas une recherche masquée du pardon, il semblerait possible d'appréhender la monumentalisation contemporaine du retour comme une mise en scène qui exprimerait un sentiment de culpabilité dissimulé par le souvenir du rituel attribué aux acteurs compromis dans la traite. D'après un autre de mes interlocuteurs, les "grands commanditaires" des razzias et des ventes n'avaient probablement ressenti aucune pitié pour le sort de la masse anonyme des esclaves, mais, "les hommes du commun", impliqués matériellement dans le transport des captifs, n'étaient sans doute pas insensibles à leur souffrance, ne serait-ce parce qu'ils en furent les acteurs directs et, ce faisant, les premiers témoins. En réfléchissant sur ces interprétations, il semble possible d'avancer l'hypothèse que, de nos jours, sur les sites où se rejoue mentalement le drame de l'histoire de l'ancienne Côte des Esclaves, de nombreux individus puissent s'identifier à ces "hommes du commun" ayant provoqué le déchirement des captifs et assisté à ses manifestations.

Sur la scène patrimoniale contemporaine, les acteurs du deuil mémoriel peuvent être les descendants des vendeurs d'antan qui prennent la parole pour les vendus d'autrefois. Cette identification relève d'une problématique discursive "paradoxale" destinée, certes, à ne pas avoir de dénouement logique, mais quand même pourvue d'une raison : celle de présentifier le passé à travers l'appropriation éthique de gestes rituels sur les lieux de l'épopée. Selon Martin Kakanacou - l'un des descendants du lignage d'origine aboméenne responsable, dans le village de Zoungbodji à l'époque de la traite, du marquage au fer rouge et du transfert, de Ouidah à la plage, des captifs dans les bateaux négriers -, l'actuel Arbre de l'Oubli, planté lors de Ouidah 92, représente l'ancien arbre autour duquel on faisait tourner les captifs pour les empêcher de "poser des problèmes " (communication personnelle).

\section{Mémoires différées des lieux de l'histoire}

À Ouidah et Abomey, une comparaison des diverses versions des lieux de mémoire semble indiquer que les interprétations des procédures inhérentes à la déportation esclavagiste varient sensiblement. À Abomey, en matière d'oubli et de retour, dans les récits que j'ai recueillis, on est relativement loin de l'entreprise «diasporique " produite par l'institution de mémoires patrimoniales censées pouvoir satisfaire, comme à Ouidah, la requête morale des Afro-Américains lors de leurs retours " touristiques ". Dans l'ancienne capitale du royaume, les lieux évoquant à la fois la 
puissance militaire aboméenne, la domination de la dynastie royale et leur implication dans la traite sont des espaces d'interprétations d'un passé moins "politiquement correct». D'après Bachalou Nondichao, ancien interprète de Pierre Verger, "personne-ressource " consultée par presque tous les chercheurs et les fonctionnaires travaillant à Abomey sur le patrimoine culturel et ethnologique dans la région, la vulgate mémorielle mise en scène sur la côte relève, en grande partie, d'une invention. À Ouidah, en revanche, le récit de Martin Kakanacou illustre le thème véhiculé par les récits contemporains relatifs à la fabrication d'un oubli qui aurait précédé une sorte de promesse faite aux captifs avant leur voyage de damnation. Cette promesse consistait à présager et préparer leur retour spirituel, après leur mort dans l'outre-monde de leur voyage. Lorsque je lui ai demandé son avis sur la séquence rituelle allant de l'injonction à oublier leur origine à l'activation d'un imaginaire d'un retour dont les esclaves auraient été les destinataires, Martin Kakanacou a remarqué : «ils [ses aïeux] étaient obligés de faire leur travail et de vaincre toute résistance de la part des esclaves». En évoquant la possibilité qu'il ne s'agissait que d'une technique de maîtrise des corps, qu'on ne "parlait" donc aux esclaves ni d'oubli ni de retour, il accepterait donc le caractère a posteriori de sa reconstruction mythique. À cet égard, la formule «parler chimiquement " qu'il utilise dans son évocation de possibles pratiques hallucinogènes ou incantatoires imposées aux captifs dans les rituels de production de l'amnésie me semble comme étant à la fois significative et suggestive. Le souvenir d'une logique inexplicable (en quelque sorte inénarrable) du sortilège s'inscrit alors dans le processus de valorisation patrimoniale de l'épopée esclavagiste devenue un héritage «immatériel » aux multiples facettes et définitions morales.

Dans un contexte traversé de récits, aux origines et aux destinations variées, l'oubli peut devenir la figure d'une dépossession, comme l'indique un entretien réalisé par Émile Désiré Ologoudou, sociologue et dignitaire du culte d'origine yoruba oro, auprès de l'un des deux chefs de cultes vodun de Ouidah, daagbo Hunon Huna $\mathrm{II}^{16}$. Selon ce témoignage que celui-ci m’a par la suite confirmé, nous sommes dans une interprétation différente de celle d'une cérémonie destinée à provoquer une amnésie, car, la place ma ja - où, lors des opérations de lancement de la Route de

16. Cf. Émile Désiré Ologoudou [s. d.]. En 2006, à Ouidah, à la suite du décès de l'ancien daagbo Hunon, un conflit a opposé les factions soutenant les deux candidats à sa succession. Trois ans plus tard, le fils du daagbo Hunon décédé que les autres dignitaires avaient empêché d'accéder au "trône", en 2006, a été proclamé à son tour daagbo Hunon Huna II par ses partisans. Ce dédoublement d'investiture n'est pas du tout exceptionnel dans un territoire où historiquement, comme le dit un adage dahoméen, «le pouvoir se dispute». 
l'Esclave, fut planté l'Arbre de l'Oubli - aurait été, durant la déportation des convois des captifs au moment de la traite, le lieu de la démence, l'endroit où les esclaves enchaînés et en colère perdaient tout à la fois leur raison (leur mémoire ?) et leurs fétiches, dont ils devaient se dessaisir au profit du daagbo Hunon de l'époque ${ }^{17}$. Le nom de cette place, ma ja, serait d'ailleurs la contraction de l'expression majeto lee ja («les enragés sont arrivés »). Une telle reconstitution, qui confirme l'implication des hiérarchies vodun dans les opérations de la traite, s'appuie sur une expression toponymique fon indiquant l'espace où est actuellement installé l'Arbre de l'Oubli, avec sa statue de Mami Wata. Elle a été transmise d'une manière partiellement différente par feu daagbo Hunon Huna lui-même à une autre chercheuse, Dana Rush, à qui le dignitaire avait confié que la rencontre rituelle entre le daagbo Hunon de l'époque et les captifs avait lieu avant leur vente aux enchères. Au cours de leur passage dans sa maison, certains captifs pouvaient "prier" leurs vodun et ceux qui montraient des qualités de spécialistes en matière de possession ou de certains savoir-faire rituels, pouvaient être retenus, mis au service des rois d'Abomey et donc sauvés de la déportation (Rush 2001) ${ }^{18}$.

Passer du souvenir d'êtres humains égarés ou en furie à la notion d'oubli, qui leur aurait été rituellement imposé, relève d'une transformation sémantique qui a peut-être été rendue possible par les métamorphoses successives de l'appréhension morale du sens de la perte de soi dont les esclaves furent victimes. À l'épreuve des interprétations contemporaines de la réalité historique de la déportation esclavagiste, la notion d'oubli finit par raconter sur la scène patrimoniale la dépossession d'une mémoire protectrice détenue par les objets que les captifs devaient remettre à leurs razzieurs de l'époque. Néanmoins, pour les responsables béninois de la valorisation de la Route de l'Esclave que j'ai rencontrés, la question de la relation entre oubli et retour se pose en termes éminemment culturels et non généalogiques. Cette logique patrimoniale confirme, à l'échelle locale et nationale béninoise, ce que, s'agissant des mémoires de l'esclavage au Ghana, Katharina Schramm (2007) souligne à juste titre : l'Unesco traite l'histoire comme un fait culturel et non comme une puissante force politique s'exerçant sur le présent.

17. "C'est à cet endroit qu'autrefois ils faisaient trois fois le tour de l'Arbre d'adieu avant de remettre dans les mains du pontife du Vodun, DAAGBO HOUNON [sic] le ou les Vodun qu'ils avaient en leur possession" (Ologoudou [s. d.]).

18. Ce récit pourrait aussi montrer l'importance logistique de la maison du daagbo Hunon dans les diverses étapes du trajet des convois de captifs, afin de justifier son intégration éventuelle dans l'itinéraire officiel de la Route de l'Esclave. 
Au cours d'un entretien avec Rachida de Souza, sociologue et membre du comité d'experts chargés par l'État béninois d'élaborer un nouveau projet d'inscription de la Route de l'Esclave sur la liste de l'Unesco, lorsque j'ai fait part de mes interrogations sur les figures de l'oubli et du retour, surprise, elle a d'abord esquissé, sans la terminer, cette réponse « en tant qu'anthropologue, vous devriez savoir..." ", sous-entendant peut-être : "vous devriez savoir...» que des logiques contradictoires peuvent cohabiter dans une narration orale. Or, la suite de son discours exprimait plutôt la nécessité d'adhérer aux contraintes d'une gouvernance patrimoniale s'attachant à fonder une histoire originale et exprimant un relativisme herméneutique et cognitif, gage d'une différence culturelle à archiver. Suivons le récit de sa reconstitution de certaines étapes du

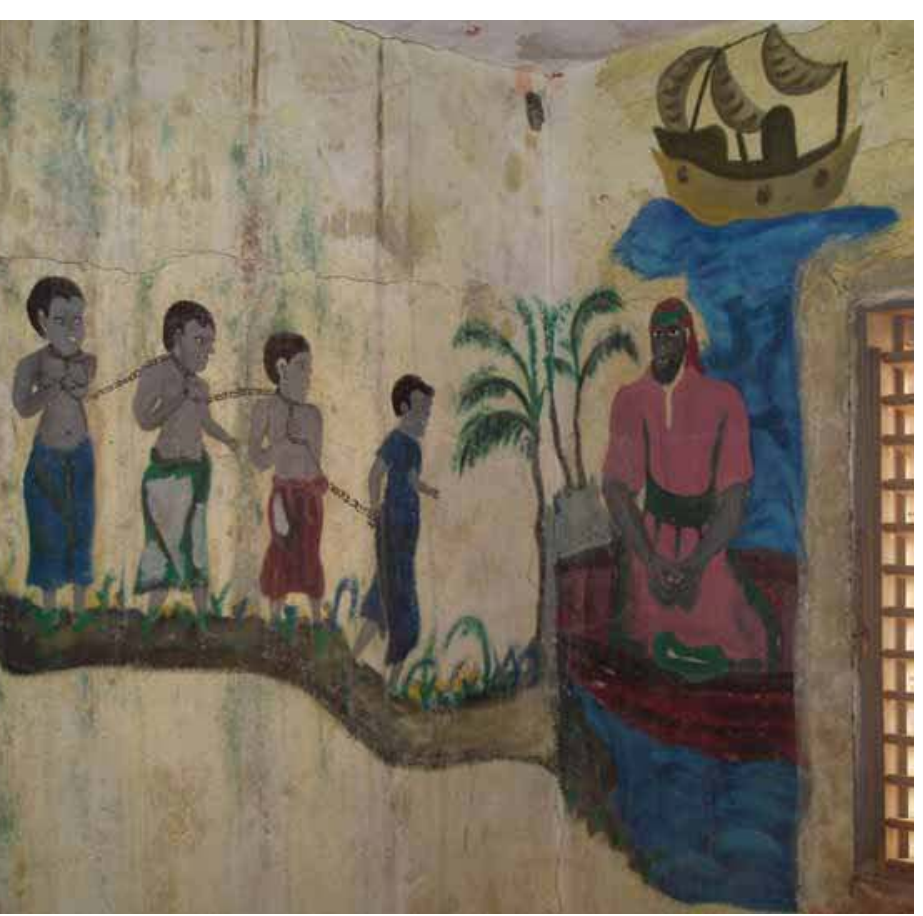

Concession de la famille Tchiakpè, ancien lieu de transit des convois d'esclaves avant leur embarquement, Ouidah, Bénin (cl. Gaetano Ciarcia, 20 I2). parcours des esclaves: à Savi, à huit kilomètres environ de Ouidah, la «cargaison était remise en forme» dans la forêt de Yênouzun, à la suite de quoi les convois arrivaient en ville en transitant par le marché Kpassé, les autels du Agadja Lègba et du Guézo Lègba ${ }^{19}$. Les captifs étaient donc emmenés dans les diverses concessions où "on les préparait» à la vente aux enchères. Le dispositif esclavagiste aboméen utilisant deux types de pouvoir, militaire et religieux, la dépossession rituelle de la force des esclaves "pérennisait» (stabilisait ?) l'emprise physique que les convoyeurs exerçaient sur eux. Selon Rachida de Souza, ceux qui étaient impliqués dans les opérations de la déportation et de la vente, en croyant aux forces occultes, pensaient que
l'«âme» ne meurt pas et que la personne du captif pouvait se venger car elle était en "connexion spirituelle» avec les divinités claniques. Tandis que la place aux Enchères transformait le corps du captif en marchandise, le passage autour de l'Arbre de l'Oubli aboutissait à une

19. C’est-à-dire les autels consacrés aux vodun Lègba des rois aboméens Agadja et Guézo. 
perte de personnalité et d'identité sociale grâce à des procédés de dépossession, qui n'était jamais intégrale. Les rituels accomplis autour de l'Arbre $\mathrm{du}$ Retour exprimaient alors la crainte des convoyeurs/vendeurs face à une malédiction possible, que leurs victimes, dont l'esprit était susceptible de revenir post mortem, pouvaient leur lancer, venant les hanter et les harceler.

Il est intéressant de noter qu'au cours de notre entretien, Rachida de Souza a insisté à plusieurs reprises sur l'idée que "la notion d'authenticité est une notion terrible" impliquant, pour les responsables de la valorisation culturelle des lieux, de faire en sorte que «les choix patrimoniaux aient une résonance dans l'imagination et le quotidien locaux ". D'après mon interlocutrice, cette perspective était désormais en accord avec les bienfaits heuristiques de la notion de "patrimoine immatériel». Ainsi rappelait-elle le principe d'une différence culturelle à la lumière de laquelle il faudrait comprendre la relation mémorielle entre oubli et retour sur la Route de l'Esclave. Pour motiver son propos, elle s'appuyait sur la distinction qu'aurait posée l'anthropologue français Bernard Maupoil (1943) dans La Géomancie à l'ancienne Côte des Esclaves. Dans ce texte, en reprenant des principes appris par son informateur principal Guèdègbe, Maupoil explique que, entre les différentes parties du corps, il y aurait une dissociation à faire entre "personnalité" (qui a été supprimée autour de l'Arbre de l'Oubli) et $s \varepsilon$, l'"âme " qui, toujours selon Rachida de Souza, aurait la possibilité de revenir grâce aux rituels accomplis autour de l'Arbre $\mathrm{du}$ Retour lors de la déportation des esclaves ${ }^{20}$. Si le $s \varepsilon$ ne peut être capturé, la qualité ultime de l' "âme » révèle l'impuissance du vendeur et explique sa quête liturgique d'un "antidote " pour contrer cette force qui s'échappe de la dépossession des corps. D’après Rachida de Souza, une telle interprétation expliquerait l'absence (je l'avais remarquée lors de mes enquêtes) de la référence au retour dans les récits recueillis à Abomey: dans l'ancienne capitale royale, les rituels avaient pour but essentiellement de veiller au bon acheminement des convois vers la côte, donc à neutraliser physiquement les captifs, alors qu'à Ouidah, les rituels auraient intégré la question morale des usages liturgiques de leurs âmes. Fondée apparemment sur une approche "endogène " du passé de la traite, cette version des faits peut être aussi analysée comme une narration susceptible de concilier le passé de l'esclavage sur le sol béninois avec une vulgate $a$ priori bonne à penser pour des rhétoriques patrimoniales globalisées,

20. Dans le chapitre intitulé «Les Âmes et le Destin », Bernard Maupoil met au jour les controverses interprétatives parmi ses informateurs au sujet d'une distinction entre les diverses parties et fonctions de l'âme dans la pensée fon; en reconnaissant qu' «en ce domaine, plus qu'en tout autre, l'accord est irréalisable» (1943: 378), il fera suivre les propos recueillis par le jugement de Guèdègbe confirmant la réalité d'une dimension quadruple de l'âme qui serait régie par un principe matriciel immortel, le $s \varepsilon$, qui «survit à l'homme, et se retrouve sur terre après la mort » (Ibid. : 389). 
sensibles à l'existence (réelle ou potentielle) de flux de groupes diasporiques qui, lors de leur "retour" en Afrique, peuvent retracer l'itinéraire physique et symbolique qui aurait été parcouru par leurs ancêtres. Cependant, la logique ici décrite peut aussi laisser sceptiques les «pèlerins » d'une mémoire perdue et à retrouver. Par exemple, lors de nos entretiens, Nouréini Tidjani-Serpos a rappelé ses visites sur la Route de l'Esclave avec des Afro-Américains qui trouvaient difficilement compréhensible, du point de vue de la véridicité historique, l'association entre oubli et retour. Selon lui, cette impossibilité à partager symboliquement un tel récit était due au fait que "dans la raison africaine le retour du mort n'est pas illogique, les cultes traditionnels n'ayant pas de paradis, ni d'enfer, ni de purgatoire». À ce propos, il remarquait : "l'Arbre du Non-Retour physique peut devenir l'Arbre du Retour-souffle, mais il faut expliquer cela aux Américains! ». Une vision cyclique de l'existence et de son au-delà se confronterait-elle à une démarche visant à réparer les maux du passé ? Une supposée ontologie africaine s'opposerait-elle donc à une téléologie afro-américaine en quête d'ancrages spirituels ? Toutefois, du point de vue de l'analyse de la commémoration patrimoniale d'une épopée comme celle mise en scène sur la Route de l'Esclave, la raison d'une telle dissociation se trouve peut être ailleurs. En ce qui concerne l'apparente incohérence du lien mémoriel, partagé entre oubli et retour, raconté et donné à voir sur et par la Route, la question se pose moins en termes de croyance en une vision cyclique restituant les morts à la terre ancestrale, qu'en termes de reconstitution mythique ritualisée d'un itinéraire. La promesse de retour faite aux âmes des captifs semble suggérer l'existence d'un "pacte " entre esclaves et ceux qui étaient chargés de la gestion concrète de leur convoyage, mais un tel pacte est difficile à comprendre selon une perspective qui n'est pas éminemment symbolique ; perspective symbolique, fautil le rappeler, que les responsables institutionnels béninois revendiquent désormais, de manière implicite ou explicite, au nom du principe patrimonial de l'immatériel. En revanche, pour de nombreux Afro-Américains, l'idée de cette transaction rituelle qui se serait passée juste avant la déportation est inacceptable, non seulement logiquement mais aussi (et je dirais surtout) moralement. En ce sens, contrairement à des personnalités institutionnelles comme Rachida de Souza et Nouréini Tidjani-Serpos, les entrepreneurs contemporains des mémoires de l'esclavage, tels Honorat Aguessy et Éric Gbodossou ${ }^{21}$, ont saisi l'efficacité performative de ce que

21. Comme nous l'avons déjà vu, Honorat Aguessy est un sociologue, fondateur à Ouidah de l'espace Zomachi et de l'IDEE (Institut de développement des échanges endogènes), ainsi que le promoteur, depuis 1998, de la Marche du repentir, devenue par la suite la Marche du devoir.../... 
l'itinéraire officiel de la Route de l'Esclave, avec sa Porte du Non-Retour, risque d'occulter: la nécessité de rendre visible hic et nunc la possibilité d'un retour physique et "spirituel» (pas seulement symbolique) conjugué à un rachat de l'identité perdue, mis en scène à travers des rituels publics contemporains (mais non pas à travers l'évocation d'un improbable rite de passage originel) ${ }^{22}$. Le sentiment de la perte doit alors s'inscrire en une matrice commune, fournie par la religion ancestrale, où l'oubli se métamorphose en un lieu commémoratif. À propos de cette relation antagoniste entre les thèmes du retour et du non-retour, il faut retenir que dans l'espace environnant son musée de la Diaspora, l'ONG ProMeTra a fait planter un troisième arbre: l' "Arbre du Souvenir», autour duquel une guide touristique, déclamant avec emphase un "message " de paix et de réconciliation, demande aux visiteurs du lieu de tourner. Il s'agit là de récitations qui expriment - tout comme Zomachi s'opposant à Zomaï (voir supra) - un complément à l'histoire racontée sur la Route de l'Esclave ${ }^{23}$. Prolongeant jusqu'à nos jours la possibilité d'un retour sans oubli, l'«Arbre du Souvenir» et la "Colonne de la Liberté» installés par ProMeTra autour de son musée fonctionnent comme un contrepoint critique par rapport à la dette d'espoir diasporique que le tracé institutionnel semble contracter, en imaginant un oubli qui aurait préparé le retour d'âmes amnésiques ${ }^{24}$. À Ouidah, comme à Accra au Ghana, ou à Gorée au Sénégal, les initiatives contemporaines qui mettent en relation le non-retour des esclaves d'antan avec le retour de leurs descendants témoignent d'une logique créatrice qui permet à une imagination spatiale et temporelle de s'exprimer. À cet égard, et après avoir été témoin sur la

[Suite de la note 21] de mémoire et du repentir. Éric Gbodossou est un médecin et psychiatre installé au Sénégal, fondateur et président de l'ONG ProMeTra (cf. infra), présente dans une dizaine de pays, parmi lesquels la France et les États-Unis.

22. À ce propos, de son côté, Tidjani-Serpos considère que la thématique du retour est bien présente sur la Route de l'Esclave. Au cours d'un entretien, il a ainsi évoqué la signification d'une partie de la scénographie de l'itinéraire qu'il a conçu : « le vent venant de la mer s'engouffre à travers la Porte et la Porte oriente ce vent revenant avec ses morts vers le mémorial de Zoungbodji ».

23. Toujours dans l'espace de Zomachi, Honorat Aguessy a fait installer une sculpture représentant l'Arbre Pini commémorant un haut lieu de la genèse de la révolution haïtienne en 1799.

24. Cette insistance sur la thématique du retour est particulièrement visible dans les itinéraires dits de la "connexion spirituelle» que ProMeTra organise au Bénin et qui s'adressent en même temps à des "pèlerins" afro-américains et à des visiteurs n'ayant pas d'origines africaines. En 2011, j’ai pu suivre un groupe d'une vingtaine de personnes en quête d'une "connexion spirituelle ", d'après l'intitulé du programme «La voie du souvenir et de la connexion spirituelle». Durant un peu plus d'une semaine, de nombreuses rencontres avec des dignitaires et des notables, des cérémonies commémoratives du passé de l'esclavage, ainsi que des séances de divination, de transe et de guérison étaient organisées en conjuguant mémoires de l'esclavage et pratiques dites vodun. Une telle conjonction est illustrée par le livret publié par ProMeTra, Les Voies du souvenir. Itinéraires des esclaves dans le Golfe du Bénin [s. d.]. Sur ProMeTra, cf. aussi : Emmanuelle Simon (2003). 


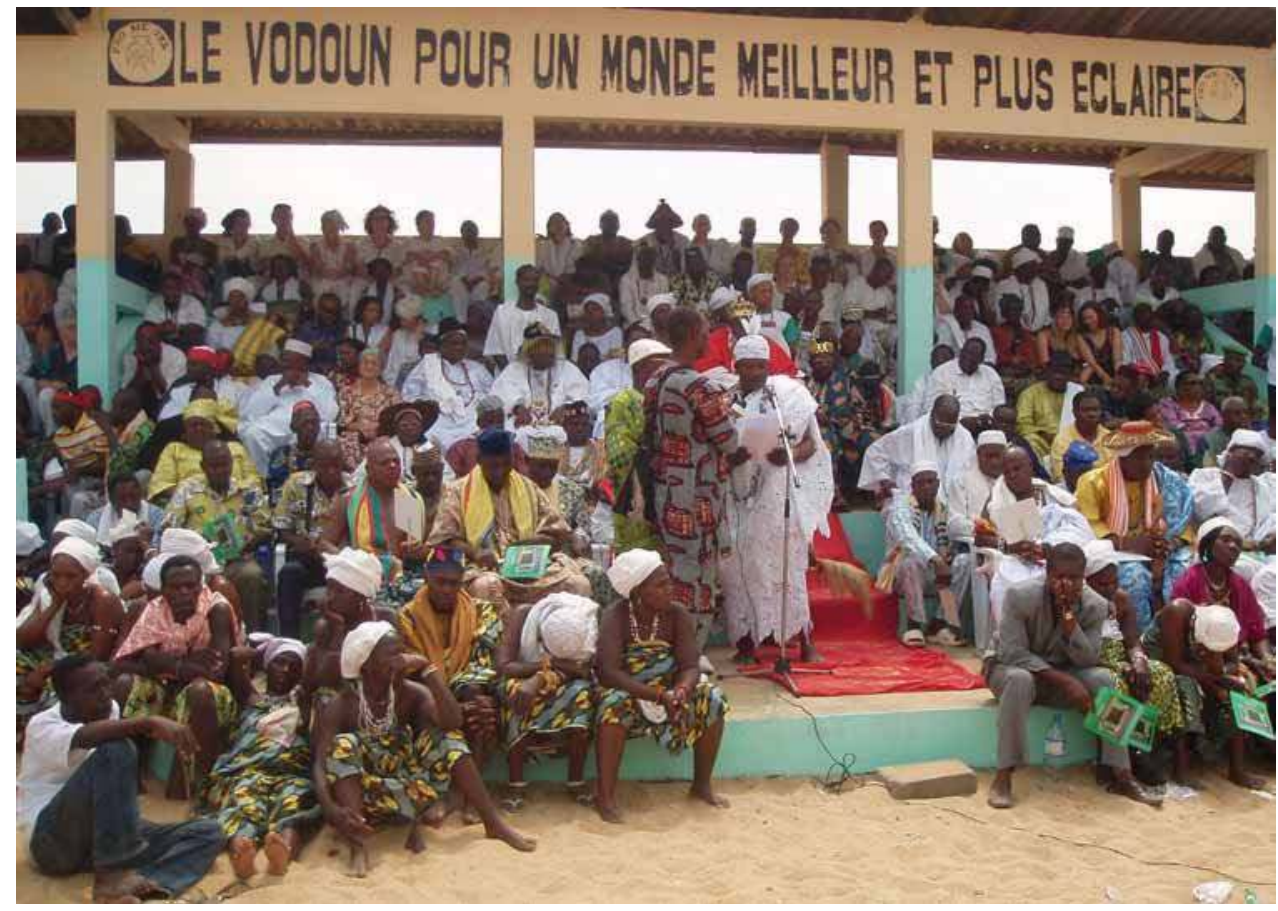

Fête nationale des religions endogènes (ancienne Fête nationale du vodun), Grand Popo, Bénin (cl. Gaetano Ciarcia, 20 I I).

plage de Ouidah de la première journée de la célébration officielle de la Fête nationale du vodun, le 10 janvier 1997, Peter Sutherland a mis l'accent sur les différentes perceptions de l'histoire de l'esclavage chez les chefs de cultes et les visiteurs afro-américains qui étaient présents. Au cours de cet événement, les dignitaires du vodun, à travers leurs discours et leurs pratiques rituelles, représentaient les esclaves comme des ancêtres sacrés. En ce sens, ils redéfinissaient l'océan comme un espace mythologique façonné par la migration d'esprits et par le retour actuel des frères de la « diaspora ». Mais, d'après le témoignage de Peter Sutherland, les visiteurs venus d'Amérique cherchaient en vain dans les actions et les paroles de leurs hôtes la conscience d'une purification des fautes relatives à l'implication des Africains dans la traite (Sutherland 1999). La performance (en tant que spectacle et interprétation théâtrale) des origines, à l'œuvre dans ce genre de commémorations à vocation " diasporique ", semble illustrer le clivage entre l'histoire des identités et la problématique anthropologique relative à leur devenir. À travers les représentations contemporaines transformant les esclaves d'autrefois en messagers planétaires du vodun et en «revenants» actuels, porteurs potentiels de développement, l'invention religieuse déploie toute sa polysémie. D'ailleurs, si les figures de l'histoire 
peuvent devenir sans cesse des métaphores mythiques aptes à exercer une fonction et un sens politiques, les mémoires rendues publiques du passé de l'esclavage participent d'un travail d'identification entre les itinéraires tragiques du passé et leur mise en inventaire. Nous sommes alors confrontés, comme l'a fait remarquer Paulla Ebron (2000), au devenir d'une condition liminale des identités en jeu qui s'étale et se décline sur plusieurs siècles.

De manière involontaire et significative, les figures de l'oubli et du retour semblent alors symboliser le hiatus qui existe entre des mémoires locales marquées par l'action latente d'un oubli nécessaire et la tentative souvent vaine d'associer les représentants de la diaspora à une histoire qui, malgré ses monuments et ses étapes rituelles commémoratives, demeure non mémorable, parce que trop sensible. À cet égard, Tidjani-Serpos a évoqué la réplique péremptoire "maintenant vous aussi vous parlez ?", par laquelle, encore aujourd'hui, pendant les conseils de famille, les représentants des lignages dominants peuvent faire taire les revendications des membres issus des anciens lignages serviles en rappelant à ceux-ci leur origine inférieure, affranchie mais non pas oubliée. Dans un tel système toujours actif de références hiérarchiques, les statues représentant les rois d'Abomey ont bien un lien avec les mémoires contemporaines de l'esclavage, en dépit des doutes émis par un chercheur de l'École du patrimoine africain (EPA) de Porto-Novo : «à l'occasion de Ouidah 92, de grandes sculptures ont été implantées tout au long de la Route, mais dont le rapport avec la traite négrière ne paraît point évident " ${ }^{25}$. Au contraire, ces sculptures matérialisent et donnent à voir ce que l'économie morale du passé suscitée par l'Unesco a tendance à négliger: le fait que le passé local de l'esclavage fait surface en se métamorphosant dans l'actualité patrimoniale et politique du contexte, sans pourtant adhérer forcément aux rhétoriques internationales officielles prônant une pacification et une démocratisation de l'histoire.

25. Cf. Edouard S. Koutinhouin (2007: 86). Instituée par l'Unesco à Porto-Novo au Bénin, l'EpA a été créée en 1998, à la suite d'une convention signée entre l'ICCROM (Centre international d'études pour la conservation et la restauration des biens culturels) et l'Université nationale du Bénin. Un des objectifs de l'EPA, qui prend le relais du programme de préservation du patrimoine Prema (Prévention dans les musées africains) lancé en 1986, est l'élargissement d'un réseau de professionnels africains capables d'assurer la conservation des collections des musées africains au sud du Sahara dans les aires francophones et lusophones. Aujourd'hui, l'EpA est une école supérieure et un centre spécialisé dans la conservation des biens culturels à la fois menacés de dégradation et susceptibles d'une valorisation. Elle participe également du travail de consultance et d'expertise inhérent à la réalisation du projet de demande d'inscription de la Route de l'Esclave sur la liste du patrimoine mondial de l'Unesco. 


\section{Le retour de l'oubli, notes conclusives}

II4 En s'interrogeant sur la pertinence «collective» de la notion d'oubli, Yosef Hahyim Yerushalmi écrit ) :

«Strictement, les peuples et les groupes ne peuvent oublier que le présent, pas le passé. En d'autres termes, les individus qui composent le groupe peuvent oublier des événements qui advinrent dans leur propre existence ; ils ne sauraient oublier le passé qui leur est antérieur [...]. Un peuple ne peut jamais "oublier" ce qu'il n’a pas d'abord reçu " $(1988: 11$ sq).

Dans le prolongement de cette considération, Jocelyne Dakhlia remarque qu' " [on] ne peut littéralement "s'oublier" que ce que l'on a soi-même connu : les sociétés et les peuples ne connaîtraient pour leur part que des "ruptures de la transmission entre générations" " (1990: 6 sq.). Néanmoins, Jocelyne Dakhlia (Ibid. : 304) reconnaît dans l'oubli la possibilité d'intégrer un agir collectif et, pour les vaincus, celle de façonner une résistance au pouvoir de ceux qui voudraient aussi imposer leur domination mémorielle, ce qu'elle appelle un "oubli sélectif». Les récits autour des Arbres sur la Route de l'Esclave que nous avons recueillis semblent confirmer que si l'oubli ne peut pas être institué ou mis en commun, un oubli sélectif peut prendre une forme scénographique. Ainsi, l'agencement d'un itinéraire patrimonial, qui vise à retracer le parcours tragique menant de l'Arbre de l'Oubli à celui du Retour, se fonde également sur un serment ancien, en l'occurrence sur une promesse de "retour", permettant aujourd'hui à l'histoire de se racheter sous forme de mythe. Pour les pèlerins de la mémoire et les visiteurs d'aujourd'hui, le retour serait la figure écran nécessaire du seul partage collectif possible de l'oubli, celui du présent. Mais, comme le remarquait Claude Lévi-Strauss (1983: 253 sq.), dans son analyse comparative des mythes amérindiens hidatsa et grecs, l'oubli " formerait système avec le malentendu, défini comme défaut de communication avec autrui, et avec l'indiscrétion, définie comme excès de communication, aussi avec autrui ». Selon cette perspective, "le motif de l'oubli sert à fonder des interdictions ou des prescriptions rituelles» (Ibid. : 258); il rendrait également compte de «l'origine de lieux dits, qui se suivent dans l'espace de façon comparable aux célébrations rituelles pendant l'année. Car les rites fixent des étapes du calendrier, comme les lieux-dits celles d'un itinéraire. Ils meublent les uns l'étendue, les autres la durée" (Ibid.). Ici, Lévi-Strauss semble prolonger sa réflexion sur l'opposition entre "rites historiques ou commémoratifs" et "les rites de deuils" (1962: 313 sq.) au sein de laquelle les premiers "recréent l'atmosphère sacrée et bénéfique des temps mythiques", alors que les seconds assureraient "la reconversion, en ancêtres, d'hommes qui ont cessé d'être des vivants ». Une telle opposition suggérerait alors comment, sur la Route de l'Esclave, les interprétations 
contemporaines de l'oubli et du retour participent de la tentative de faire cohabiter dans l'espace les principes de la réversibilité et de l'irréversibilité du temps. Il serait possible d'avancer l'hypothèse que dans ce dispositif chronotope, le deuil des humains morts pour de vrai (les esclaves) intègre et prédit, à travers leur personnification diasporique contemporaine, la mémoire de leur retour en tant qu' « héros mythiques " ${ }^{26}$.

Si le rituel est la scène d'une quête collective d'une continuité qui se réalise grâce à une expérience mythique toujours à renouveler, si le rituel s'oppose donc à l'oubli, cela est possible grâce à l'alternance entre un excès de communication avec autrui et la réserve secrète de symboles toujours bons à être repensés et réinterprétés. Un flux de paroles imagées (ou fictionnelles) véhiculerait ce qui du passé doit être oublié, tu ou, au contraire, célébré. Comment alors comprendre la place de l'oubli comme propédeutique du retour dans l'itinéraire ritualisé d'une épopée tragique comme celle racontée par la scénographie de la Route de l'Esclave à travers l'invention narrative de ses Arbres?

À partir de l'exemple athénien, Nicole Loraux s’interroge : entre le vœu d'un oubli nécessaire à la continuité pacifique de la vie sociale et l'interdiction d'un souvenir conflictuel, serait-il possible de penser l'émergence d'une histoire marquée par le concours de ces deux injonctions indispensables à la cité : une revendiquant l'amnésie et l'autre empêchant tout effort mémoriel ? Sur l'activité mémorielle du passé de l'esclavage dans le Bénin contemporain, nous pouvons nous poser une question comparable.

En octobre 2005, au tout début de mes enquêtes au Bénin, pendant un entretien avec un notable de Ouidah, j'avais été frappé par une formule hermétique et paradoxale répétée au moins à deux reprises: "l'oubli oui, le pardon non». Cette formulation était faite par une personnalité d'origine yoruba, c'est-à-dire issue d'une communauté minoritaire à Ouidah dont la plupart de ses membres sont des descendants d'anciens esclaves, non seulement d'esclaves déportés en Amérique, mais aussi d'esclaves employés sur place jusqu'au début du XXe siècle dans les maisons et dans les plantations de palmiers à huile. Elle m'était apparue significative de la relation controversée entre des mémoires collectives formellement apaisées et les souvenirs, très différents, selon les individus et les familles, concernant l'héritage d'un passé esclavagiste qui a affecté d'une manière cruciale l'histoire sociale de la ville. "L'oubli oui, le pardon non", ne saurait être une formule synthétique des sentiments les plus antinomiques suscités par le passé de l'esclavage chez d'autres membres de la communauté yoruba ou d'autres communautés connotées par la

26. Cf. Claude Lévi-Strauss (1962) ; je remercie Jean Jamin de m’avoir rappelé cette référence. 
condition servile d'autrefois. Révélant un usage politique et identitaire de l'oubli, l'énoncé en question ne serait pas la manifestation de l'incapacité à pardonner mais celle de la réversibilité assumée de tout acte mémoriel : son immanence dans les pratiques sociales qui participent à la fois de l'érosion des faits du passé et de leur mise en jachère. Si une telle assertion nous parle du fait que "l'affranchissement est un secret " (Meillassoux 1998 : 122), elle exprime également les flottements et les silences impliqués, ainsi qu'une opposition latente à la rhétorique officielle fondée sur la nécessité du souvenir et de la réconciliation qui ont marqué la mise en patrimoine de l'histoire de l'esclavage à Ouidah. L'oubli devient alors le masque social de l'affranchissement dont le rappel doit être aboli. La condition présente est censée oblitérer l'état servile d'autrefois. Comme l'a fait remarquer Claude Meillassoux :

«[...] les véritables affranchis, c'est-à-dire les esclaves ayant récupéré toutes les prérogatives et l'honneur des francs, on ne peut les nommer, ni même admettre qu'on les connaît comme tels, sans leur faire perdre aussitôt le bénéfice de la franchise dont l'objet est précisément d'effacer à jamais le stigmate originel de la capture ou de la naissance servile» (Ibid).

Cette logique entre implicitement en conflit avec un événement comme Ouidah 92 et la réalisation de la Route de l'Esclave ; au sein de ces espaces présentifiant le passé comme ressource culturelle et politique, il nous est possible d'observer à l'œuvre ce que Nicole Loraux (1997: 165) définit comme la persistance du "non-oubli». Si l'Arbre du Retour participe de l'époque patrimoniale du rapprochement diasporique, en imaginant une promesse qui aurait été faite aux esclaves avant leur déportation, l'efficacité d'une telle rhétorique est soumise à son intégration dans un dispositif mémorial ${ }^{27}$ ritualisé qui, à travers les étapes autour de l'oubli et du retour, convertit magiquement «la colère en gloire» (Ibid.: 171). Ainsi, nous pourrions imaginer que dans l'épopée contemporaine, autour de l'Arbre $\mathrm{du}$ Retour, les fantômes des esclaves qui transitèrent par le lieu dit majeto lee ja («les enragés sont arrivés»), devenu aujourd'hui la place de l'Arbre de l'Oubli, reviennent en ancêtres pacificateurs et aménageurs de patrimoine culturel, figures mythiques d'une amnésie qui se commémore.

Université Paul-Valéry-Montpellier 3, Centre d'études et de recherches comparatives en ethnologie, EA 4584 - E1, Montpellier ciarcia.gaetano@wanadoo.fr

27. Ici, le mot «mémorial » est utilisé à dessein pour indiquer les supports physiques ou les notions discursives exprimant la volonté de se souvenir des faits du passé en complément de l'adjectif "mémoriel » qui dénoterait plutôt les qualités spontanées ou les capacités sélectives des activités de la mémoire. 
MOTS CLÉS/KEYWORDS : Route de l'Esclave/Slave Route - Bénin/Benin - Ouidah - mémoire de l'esclavage/memory of slavery - vodun/voodoo - épopée mémorielle/memorial epic - itinéraire rituel/ritual itinerary.

\section{BIBLIOGRAPHIE}

Augé, Marc

2001 Les Formes de l'oubli.

Paris, Payot \& Rivages.

\section{Berbain, Simone}

1942 Étude sur la traite des Noirs au golfe de Guinée. Le comptoir français de Juda (Ouidah) au XVIII siècle. Paris, Librairie Larose ("Mémoires de l'IFAN»).

\section{Ciarcia, Gaetano}

2008a «Restaurer le futur: sur la Route de l'Esclave à Ouidah, Bénin ", Cahiers d'études africaines 192: 687-705.

2008b «Rhétoriques et pratiques de l'inculturation : une généalogie "morale" des mémoires de l'esclavage au Bénin", Gradhiva nouv. sér. 8 : 28-45.

Dakhlia, Jocelyne

1990 L'Oubli de la cité. La mémoire collective à l'épreuve du lignage dans le Jérid tunisien. Paris, La Découverte.

Division du Patrimoine culturel [s. d.] Remarques du Centre du patrimoine mondial. Document interne. Cotonou, Ministère de la Culture.

\section{Ebron, Paulla}

2000 «Tourists as Pilgrims : Fashioning of Transatlantic Politics ", American Ethnologist 26 (4) : 910-932.

\section{Koutinhouin, Edouard S.}

2007 "Les enjeux de l'inscription des sites sur la Liste du Patrimoine mondial », in Toussaint Yaovi Tchitchi, ed., La Route de l'Esclave au Bénin. Actes de la table ronde du 17 décembre 2005. Cotonou, Ablodè \& Tundé : 74-87.

\section{Law, Robin}

2004 Ouidah. The Social History of a West African Slaving "Port". Athens, Ohio University.

2008 "Commemoration of the Atlantic Slave Trade in Ouidah ", Gradhiva nouv. sér. $8: 10-25$.

\section{Lévi-Strauss, Claude}

1962 La Pensée sauvage. Paris, Plon.

1975 La Voie des masques. Genève, Skira.

1983 "Mythe et oubli ", in Le Regard éloigné. Paris, Plon : 253-260.

\section{Loraux, Nicole}

1997 La Cité divisée. L'oubli dans la mémoire d'Athènes. Paris, Payot \& Rivages.

Maupoil, Bernard

1943 La Géomancie à l'ancienne Côte des Esclaves. Paris, Institut d'ethnologie.

Meerloo, Joost

1968 «Delayed Mourning in Victims of Extermination Camps ", in Henry Krystal, ed, Massive Psychic Trauma. New York, International Universities Press :

70-82.

\section{Meillassoux, Claude}

1998 [1986] Anthropologie de l'esclavage. Le ventre de fer et d'argent.

Paris, Presses

universitaires de France.

\section{Ologoudou, Émile Désiré}

[s. d.] «Interview de sa Majesté daagbo Huounon Huna II", in Culte des morts et devoir de mémoire. Les masques Egungun et Oro à Glexwé-Ouidah.

Manuscrit non paginé. 
Preston Blier, Suzanne

118
1995 African Vodun. Art, Psychology, and Power. Chicago-London, University of Chicago Press.

Promotion de la médecine traditionnelle (PRoMeTra)

[s. d.] "Les voies du souvenir ", in Itinéraires des esclaves dans le golfe du Bénin. Dakar, PROMETRA Internationale.

\section{Ricœur, Paul}

2000 La Mémoire, l'histoire, l'oubli.

Paris, Le Seuil.

Rush, Dana

2001 "Contemporary Vodun Arts

of Ouidah, Benin ", African Arts 34 : 32-47, 94-96.

\section{Schramm, Katharina}

2007 «Slave Route Projects : Tracing the Heritage of Slavery in Ghana ", in Ferdinand de Jong \& Michael Rowlands, eds, Reclaiming Heritage. Alternative Imaginaries of Memory in West Africa.

Walnut Creek, Left Coast Press («Publications of the Institute of Archaelogy, University College London ») : 71-98.

\section{Severi, Carlo}

2007 Le Principe de la chimère.

Une anthropologie de la mémoire. Paris,

Éd. Rue d'Ulm-Musée du quai Branly, (" Æsthetica») [éd. orig. : Il Percorso e la voce. Un'antropologia della memoria. Torino, Einaudi, 2004].

\section{Simon, Emmanuelle}

2003 "Une exportation du New Age en Afrique?", Cahiers d'études africaines $172: 883-898$.

Sutherland, Peter

1999 «In Memory of Slaves : An African

View of the Diasporas in the Americas ", in Jean Muteba Rahier, ed., Representations of Blackness and the Performances of Identities. Westport-London, Bergin \& Carvey: 195-211.

\section{Tardits, Claude}

1958 Porto-Novo. Les nouvelles générations africaines entre leurs traditions et l'Occident. Paris-La Haye, Mouton \& Co.

Tchitchi, Toussaint Yaovi

2007 "Postface", in La Route de l'Esclave au Bénin. Actes de la table ronde du 17 décembre 2005. Cotonou, Ablodè $\&$ Tundè.

Union générale pour le développement de Ouidah (UGDO)

1985 Les Voies de la renaissance de Ouidah. Caen, Kanta.

Yerushalmi, Yosef Hayim

1988 "Réflexions sur l'oubli », in Yosef H. Yerushalmi et al., Usages de l'oubli.

Contributions au colloque de Royaumont (1987). Paris, Le Seuil : 7-21.

Young, James E.

1993 «Écrire le monument: site, mémoire, critique ", Annales ESC 3 : 729-743. 
Gaetano Ciarcia, L'oubli et le retour: figures d'une épopée mémorielle sur la Route de l'Esclave au Bénin. - Au Bénin, depuis les débuts des années 1990, le processus institutionnel de connexion mémorielle entre le passé de l'esclavage et les pratiques cultuelles vodun a été scandé par le Festival Ouidah 92 et le lancement, sous l'égide de l'Unesco, de l'itinéraire intercontinental de la Route de l'Esclave. Les notions de l'oubli et du retour mises en exergue sur la Route par les deux Arbres éponymes montrent comment cet itinéraire, à la fois monumental et rituel, devrait permettre aujourd'hui à une histoire tragique de se "racheter" sous forme de discours mythique. Dans les diverses versions de ce mythe, où un lien mémoriel partagé entre oubli et retour est raconté et donné à voir, les récits locaux de l'esclavage font surface en se métamorphosant dans l'actualité patrimoniale et politique de la situation. De tels récits n'adhèrent pas pourtant aux diverses rhétoriques internationales et nationales, officielles et diasporiques, prônant, en quelque sorte, une pacification et une démocratisation de l'histoire.
Gaetano Ciarcia, Oblivion and Return: Figures in a Memorial Epic on the Slave Route in Benin. - Since the early 1990s in Benin, the institutional process of connecting through memorials the past of slavery and the practices of voodoo has been highlighted by the Ouidah 92 Festival and the launching, under the auspices of Unesco, of the intercontinental itinerary of the Slave Route. Two eponymous trees on this road draw attention to the concepts of oblivion and return, thus showing how, thanks to this monumental and ritual itinerary, a tragic history should be able to "redeem" itself in the form of a mythical discourse. In the various versions of this myth, which tells about and visually presents a shared link between forgetting and remembering, local accounts of slavery rise to the surface while being metamorphosed in the patrimonial and political relevance of the situation. Such accounts do not adhere to international or national forms of rhetoric, whether from officials or from the diaspora, that tend to advocate a pacification and democratization of history. 